JOURNAL OF THE

AMERICAN MATHEMATICAL SOCIETY

Volume 15, Number 4, Pages 787-815

S 0894-0347(02)00399-5

Article electronically published on June 21, 2002

\title{
DERIVED HILBERT SCHEMES
}

\author{
IONUŢ CIOCAN-FONTANINE AND MIKHAIL M. KAPRANOV
}

\section{INTRODUCTION}

(0.1) The Derived Deformation Theory (DDT) program (see [Kon, CK] for more details and historical references) seeks to avoid the difficulties related to the singular nature of the moduli spaces in geometry by "passing to the derived category", i.e., developing an appropriate version of the (nonabelian) derived functor of the functor of forming the moduli space. The resulting geometric objects are sought to be not ordinary varieties or schemes but rather dg-schemes, i.e., geometric objects whose algebras of functions are commutative differential graded (dg-)algebras and which are considered up to quasi-isomorphism. Moreover, they are expected to be smooth in the sense that the corresponding dg-algebras can be obtained from a smooth commutative algebra in the usual sense by adding projective modules of generators in each negative degree.

At the present time, one can say that the DDT program is well established in the formal case (i.e., one has a good theory of the derived version of the formal neighborhood of any point in the usual deformation space; see [BK, Hi1, Man]). However, global derived moduli spaces (of great interest for enumerative applications) are much less understood. In [CK], we have constructed the derived version of the first global algebro-geometric moduli space, namely of the Grothendieck's Quot scheme. The aim of the present paper is to give a construction (using a similar but different approach) of the derived Hilbert scheme.

(0.2) While in the usual algebraic geometry, the Hilbert scheme is a particular case of the Quot scheme, the two constructions diverge in the derived world. To see the difference, consider a smooth projective variety $X$ and a subvariety $Z \subset X$ with Hilbert polynomial $h$ and sheaf of ideals $\mathcal{I}_{Z}$. Then $Z$ represents a point $[Z]$ in $\operatorname{Hilb}_{h}(X)=\operatorname{Quot}_{h}\left(\mathcal{O}_{X}\right)$. The tangent space to the dg-manifold $\operatorname{RQuot}_{h}\left(\mathcal{O}_{X}\right)$ at the point $[Z]$ is a cochain complex with cohomology

$$
H^{i} T_{[Z]}^{\bullet} R Q \operatorname{Rot}_{h}\left(\mathcal{O}_{X}\right)=\operatorname{Ext}_{\mathcal{O}_{X}}^{i}\left(\mathcal{I}_{Z}, \mathcal{O}_{Z}\right) .
$$

In the present paper we construct a dg-manifold $R H i l b_{h}^{L C I}(X)$ whose degree 0 truncation $\pi_{0} R H i l b_{h}^{L C I}(X)$ is the open part in $\operatorname{Hilb}_{h}(X)$ consisting of locally complete intersections and whose tangent dg-space at a point $[Z]$ for $Z$ as above is given by

$$
H^{i} T_{[Z]}^{\bullet} R H i l b_{h}^{L C I}(X)=H^{i}\left(Z, N_{Z / X}\right),
$$

where $N_{Z / X}$ is the normal bundle. See (4.2) for precise statements in the case of arbitrary $Z$, when the relative cotangent complex is involved. When $h$ is identically

Received by the editors August 14, 2000.

2000 Mathematics Subject Classification. Primary 14M30; Secondary 18 G50.

(C)2002 American Mathematical Society 
1, we get $\operatorname{RHilb}_{h}^{L C I}(x)=\operatorname{Hilb}_{h}(X)=X$, but $\operatorname{RQuot}_{h}\left(\mathcal{O}_{X}\right)$ is not identified with $X$ in any way, because for $Z=\{x\}$ the tangent dg-space at $x$ to RQuot has by (0.2.1) the $i$ th cohomology equal to $\Lambda^{i+1}\left(T_{x} X\right)$.

The dg-manifolds RQuot from [CK] are suitable for the construction of the derived moduli spaces of vector bundles on a fixed algebraic variety, as explained in [CK], Remark 4.3.8. In contrast, the dg-schemes RHilb constructed here should play the same role for the derived moduli spaces of algebraic varieties themselves. For example, we use them to construct two types of geometric derived moduli spaces:

- The derived space of maps $\operatorname{RMap}(C, Y)$ from a fixed projective scheme $C$ to a fixed smooth projective variety $Y$; see (4.3.5)

- The derived stack of stable degree $d$ maps $R \bar{M}_{g, n}(Y, d)$ from $n$-pointed nodal curves to a given smooth projective variety $Y$; see Section 5 . The nonderived stacks of stable maps $\bar{M}_{g, n}(Y, d)$ were introduced by Kontsevich Kon (see also $[\mathrm{BM}],[\mathrm{FP}]$ ), and turned out to be extremely important in the mathematical theory of Gromov-Witten invariants. However, Kontsevich in fact proposed in loc cit. to construct the derived version of $\bar{M}_{g, n}(Y, d)$ as well. Our work carries out this proposal.

(0.3) Similarly to the case of the usual algebro-geometric moduli spaces, it would be nice to characterize RHilb and RQuot in terms of representability of some functors. This is not easy, however, as the functors should be considered on the derived category of dg-schemes (with quasi-isomorphisms inverted) and for morphisms in this localized category there is currently no explicit description. This issue should probably be addressed in a wider foundational context for dg-algebraic geometry, in which one should systematically work with objects glued from dg-schemes in our present sense by means of gluing maps which are only quasi-isomorphisms on pairwise intersections, satisfying cocycle conditions only up to homotopy on triple intersections, etc.

Nevertheless, our derived Hilbert scheme RHilb carries a certain natural family of commutative dg-algebras which over the usual Hilbert scheme is just the family of truncations of the graded coordinate rings of $Z \subset X$. In contrast, the dg-scheme RQuot carries a family of dg-modules, not algebras.

Accordingly, the main technical step in constructing RHilb is the construction of the derived moduli space $R C A(W)$ of structures of a commutative algebra on a given finite-dimensional vector space $W$; see Section 3 . We achieve this by using a certain dg-resolution of the operad $\mathcal{C}$ om describing commutative algebras. Derived moduli spaces of operad algebras were the subject of several recent papers [Re, [Hi2], [KS], although our approach differs from the approaches of these papers. The similar step in the construction of RQuot in [CK] involved the derived space of structures of an $A$-module on $W$, where $A$ and $W$ are a fixed algebra and vector space, respectively. That was achieved by using a dg-resolution of $A$.

(0.4) Let us now describe the content of the paper. In Section 1 we give an identification of the Hilbert scheme of a projective scheme $X$ with graded coordinate ring $A$ with the scheme of graded ideals of the finite-dimensional graded algebra (without unit) $A_{[p, q]}$ for $0 \ll p \ll q$. This result seems to be new and interesting by itself. It improves Theorem 1.4.1 of [CK].

Section 2 provides some background information on the cotangent complexes and Harrison homology. The only possibly new result here is Theorem 2.2.2 which 
connects the relative cotangent complex of a morphism of graded algebras with the relative cotangent complex of the morphism of their projective spectra.

In Section 3 we develop the central construction of the paper: the derived scheme of ideals in a finite-dimensional commutative algebra. This is parallel to (but different from) $\mathrm{CK}]$ where the corresponding role was played by the derived schemes of $A$-submodules in a finite-dimensional module over a given algebra $A$.

In Section 4 we apply the algebraic formalism of Section 3 to the truncated graded coordinate rings $A_{[p, q]}$, give the constructions of the dg-manifolds $R H i l b \frac{\leq m}{h}(X)$ and $R H i l b_{h}^{L C I}(X)$ and prove the properties stated in (0.3). We also construct the derived space of maps $\operatorname{RMap}(C, Y)$.

Finally, in Section 5 we relativize the above construction to the case of a base stack $\mathcal{S}$ of finite type. In particular, we develop the beginnings of a theory of dg-stacks. We apply this to the construction of the derived stack of stable maps.

(0.5) We would like to thank V. Drinfeld and K. Behrend who made us realize that there should be not one, but two derived versions of the Hilbert scheme. Both authors were partially supported by NSF grants.

\section{Hilbert SCheme as the VARIETy of ideals}

(1.1) Generalities on Hilb and Quot. We work over a fixed field $\mathbb{K}$. Let $X \subset \mathbb{P}^{n}$ be a projective scheme over $\mathbb{K}$ and let $\mathcal{O}_{X}(1)$ be the very ample sheaf defining the projective embedding. For a coherent sheaf $\mathcal{G}$ on $X$ we denote $\mathcal{G}(i)=\mathcal{G} \otimes \mathcal{O}_{X}(i)$ and denote by $h^{\mathcal{G}}(t) \in \mathbb{Q}[t]$ the Hilbert polynomial of $\mathcal{G}$. Thus $h^{\mathcal{G}}(i)=\chi(\mathcal{G}(i))$ for $i \in \mathbb{Z}$.

Fix a polynomial $h \in \mathbb{Q}[t]$ and let $\operatorname{Hilb}_{h}(X)$ be the Hilbert scheme parametrizing closed subschemes $Z \subset X$ such that $h^{\mathcal{O}_{Z}}=h$. For such $Z$ we denote by $\mathcal{I}_{Z} \subset \mathcal{O}_{X}$ the sheaf of ideals of $Z$ and by $[Z]$ the $\mathbb{K}$-point of $\mathrm{Hilb}_{h}(X)$ corresponding to $Z$. Note that the full Hilbert scheme of $X$ does not depend on the projective embedding, but the way it is decomposed into the disjoint union of the subschemes $H_{i l b}(X)$ does. Should we need to emphasize the dependence of these subschemes on the embedding, we will use the notation $\operatorname{Hilb}_{h}\left(X, \mathbb{P}^{n}\right)$.

More generally, if $\mathcal{F}$ is a coherent sheaf on $X$, we have the $Q u o t$ scheme $\operatorname{Quot}_{h}(\mathcal{F})$ parametrizing flat families of quotients $\mathcal{F} \rightarrow \mathcal{G}$ with $h^{\mathcal{G}}=h$. The Hilbert scheme is a particular case corresponding to $\mathcal{F}=\mathcal{O}_{X}$. See $\mathrm{Gr}$, [Kol, $\mathrm{Vi}$, for more background.

Recall Grothendieck's Grassmannian embedding of the Quot scheme; see loc. cit. Set $k=h^{\mathcal{F}}-h \in \mathbb{Q}[t]$. We will identify the polynomial $k$ with the sequence of its values $k_{i}=k(i), i \in \mathbb{Z}$. Fix $r \gg 0$. Associating to a quotient $\mathcal{F} \rightarrow \mathcal{G}$ the linear subspace $\operatorname{Ker}\left\{H^{0}(X, \mathcal{F}(r)) \rightarrow H^{0}(X, \mathcal{G}(r))\right\}$ defines a regular map

$$
\alpha_{r}: \operatorname{Quot}_{h}(\mathcal{F}) \rightarrow G\left(k(r), H^{0}(X, \mathcal{F}(r))\right) .
$$

(1.1.1) Theorem. For $r \gg 0$ the map $\alpha_{r}$ identifies Quot $_{h}(\mathcal{F})$ with a closed subscheme of the Grassmannian $G\left(k(r), H^{0}(X, \mathcal{F}(r))\right)$.

(1.2) The $A$-Grassmannian and the scheme of ideals. Let $A$ be an associative algebra over $\mathbb{K}$ (possibly without unit) and $M$ a left $A$-module which is finitedimensional (over $\mathbb{K}$ ). Then we have the $A$-Grassmannian $G_{A}(k, M) \subset G(k, M)$, parametrizing $k$-dimensional submodules $V \subset M$; see n.(1.3) of [CK]. More generally, let $A$ be a graded algebra and let $M$ be a finite-dimensional graded $A$-module. 
Then, for every sequence $k=\left(k_{i}\right)_{i \in \mathbb{Z}}$ of nonnegative integers we have the graded $A$-Grassmannian $G_{A}(k, M)$ which parametrizes graded submodules $V \subset M$ with $\operatorname{dim}_{\mathbb{K}}\left(V_{i}\right)=k_{i}$.

Suppose now that $A$ is finite dimensional. Then we can take $M=A$; the scheme $G_{A}(k, A)$ will be denoted $J(k, A)$ and called the scheme of (left) ideals in $A$ of dimension $k$. For such an ideal $I \subset A$ we will denote $[I] \in J(k, A)$ as the corresponding $\mathbb{K}$-point.

Suppose, moreover, that $A$ is commutative. Then left ideals are two-sided and for such an ideal $I$ the quotient $A / I$ is again a commutative algebra. In this case the Zariski tangent spaces to $J(k, A)$ are given by:

$$
T_{[I]} J(k, A)=\operatorname{Hom}_{A}(I, A / I)=\operatorname{Hom}_{A / I}\left(I / I^{2}, A / I\right) .
$$

Here the first equality is the general property of $A$-Grassmannians (see n.(1.3.2) of [CK]) and the second equality is due to the commutativity of $A$.

Now let $A$ be a graded finite-dimensional commutative algebra and let $k=\left(k_{i}\right)$ be a sequence of integers, as before. We then have the scheme $J(k, A)$ (the scheme of graded ideals) parametrizing graded ideals $I \subset A$ with $\operatorname{dim}\left(I_{i}\right)=k_{i}$. The description of the tangent space in this case is modified as follows:

$$
T_{[I]} J(k, A)=\operatorname{Hom}_{A / I}^{0}\left(I / I^{2}, A / I\right),
$$

where $\operatorname{Hom}^{0}$ means the space of homomorphisms of degree 0.

(1.3) Hilb as the scheme of ideals. We return to the situation of (1.1) and let $A=\bigoplus_{i} H^{0}\left(X, \mathcal{O}_{X}(i)\right)$ be the homogeneous coordinate ring of $X$. For $p \geq 0$ let $A_{\geq p}=\bigoplus_{i \geq p} H^{0}\left(X, \mathcal{O}_{X}(i)\right)$ be the truncation of $A$ in degrees $\geq p$. This is an ideal in $A$; we denote by $A_{[p, q]}=A_{\geq q} / A_{\geq p}, p \leq q$, the finite-dimensional truncation in degrees from $p$ to $q$. Both $A_{\geq p}$ and $\bar{A}_{[p, q]}$ are graded commutative algebras without unit. In particular, the construction of the graded scheme of ideals is applicable to $A_{[p, q]}$.

Suppose that $p \gg 0$. Then for any $q \geq p$ the Grassmannian embeddings $\alpha_{r}$, $p \leq r \leq q$, from (1.1) define a morphism $\beta_{[p, q]}: H i l b_{h}(X) \rightarrow J\left(k, A_{[p, q]}\right)$. Here $k$ is the sequence $\left(k_{p}=h^{\mathcal{O}_{X}}(p)-h(p), \ldots, k_{q}=h^{\mathcal{O}_{X}}(q)-h(q)\right)$.

Let us now formulate the main result of this section.

(1.3.1) Theorem. If $0 \ll p \ll q$, then the morphism $\beta_{[p, q]}$ identifies $H i l b_{h}(X)$ with the scheme of graded ideals $J\left(k, A_{[p, q]}\right)$.

To begin the proof, recall a particular case of a result proved in [CK], (1.4.1).

(1.3.2) Theorem. (a) For $0 \ll p \ll q$ the natural embedding $\alpha_{[p, q]}: \operatorname{Hilb}_{h}(X) \rightarrow$ $G_{A}\left(k, A_{[p, q]}\right)$ is an isomorphism.

(b) More precisely, (a) is true whenever $p$ is such that the map $\alpha_{p}$ from (1.1.1) is an embedding and $q$ is large enough compared with $p$.

Note that we have an embedding

$$
\phi: G_{A}\left(k, A_{[p, q]}\right) \hookrightarrow J\left(k, A_{[p, q]}\right)
$$

since an $A$-submodule is automatically an $A_{[p, q]}$-submodule. So Theorem 1.3.1 strengthens Theorem 1.3.2 which we will nevertheless use as a starting point. Also, in the proof of Theorem 1.3.1 we can and will assume that $\mathbb{K}$ is algebraically closed.

Choose $p>0$ such that $\alpha_{p}$ is an embedding and consider the Veronese subalgebra $B=\bigoplus_{j} A_{p j}$, graded so that $B_{j}=A_{p j}$. Let $\mathbb{P}^{n}=\mathbb{P}\left(A_{1}^{*}\right)$ be the ambient space of 
the initial projective embedding of $X$. Then $\mathbb{P}^{N}=\mathbb{P}\left(A_{p}^{*}\right)=\mathbb{P}\left(B_{1}^{*}\right)$ is the ambient space of another projective embedding known as the $p$-fold Veronese embedding. In other words, $X=\operatorname{Proj}(A)=\operatorname{Proj}(B)$. Note that on $X$ we have $\mathcal{O}_{\mathbb{P}^{N}}(1) \simeq \mathcal{O}_{\mathbb{P}^{n}}(p)$. The independence of the full Hilbert scheme on the projective embedding implies that

$$
\operatorname{Hilb}_{h}\left(X, \mathbb{P}^{n}\right)=\operatorname{Hilb}_{h^{\prime}}\left(X, \mathbb{P}^{N}\right), \quad \text { where } \quad h^{\prime}(t)=h(p t) .
$$

Let $r \geq 1$. Then, setting $k_{i}^{\prime}=k_{p i}$ for $i=1, \ldots, r$, we find

$$
J\left(k^{\prime}, B_{[1, r]}\right)=G_{B}\left(k^{\prime}, B_{[1, r]}\right) .
$$

By Theorem 1.3.2(b) we have that for $r \gg 0$ each of the schemes in (1.3.5) is identified with $\operatorname{Hilb}_{h^{\prime}}\left(X, \mathbb{P}^{N}\right)$, i.e., with $\operatorname{Hilb}_{h}\left(X, \mathbb{P}^{n}\right)$.

We now take $q=p r, r \gg 0$, and construct an inverse to the embedding (1.3.3). Given a graded ideal $I \subset A_{[p, q]}$, we form the ideal $J=\bigoplus_{j} I_{p j}$ in $B_{[1, r]}$ obtained by taking every $p$ th graded component of $I$. Let $\mathcal{J} \subset \mathcal{O}_{X}$ be the sheaf of ideals associated to $J$ by (1.3.4) and (1.3.5). By construction, $\mathcal{O}_{X} / \mathcal{J}$ has Hilbert polynomial $h^{\prime}$ with respect to the embedding of $X$ into $\mathbb{P}^{N}$, and therefore has Hilbert polynomial $h$ with respect to the embedding of $X$ into $P^{n}$, so it represents a point of $\operatorname{Hilb}_{h}\left(X, \mathbb{P}^{n}\right)$. Now define

$$
\psi(I)=\bigoplus_{i=p}^{q} H^{0}\left(X, \mathcal{J} \otimes \mathcal{O}_{\mathbb{P}^{n}}(i)\right) .
$$

Our theorem follows from the next lemma.

(1.3.6) Lemma. (a) $\psi(I) \in G_{A}\left(k, A_{[p, q]}\right)$.

(b) The correspondence $I \mapsto \psi(I)$ gives rise to a morphism of schemes $\psi$ : $J\left(k, A_{[p, q]}\right) \rightarrow G_{A}\left(k, A_{[p, q]}\right)$.

(c) The morphisms $\phi$ and $\psi$ are mutually inverse.

Proof. (a) It is clear that $\psi(I)$ is a graded ideal in $A_{[p, q]}$. Because of the choice of $p$, the Hilbert function of the ideal $\psi(I)$ is given by its Hilbert polynomial. (Among other things, the choice of $p$ implies this property for all ideals from $H i l b_{h}(X)$.) In other words, $\operatorname{dim}_{\mathbb{K}} \psi(I)_{j}=k_{j}$, as claimed.

(b) First of all, passing from $I$ to $J$ is clearly a morphism of schemes $J\left(k, A_{[p, q]}\right) \rightarrow$ $J\left(k^{\prime}, B_{[1, r]}\right)$. The target of this morphism is identified with $\operatorname{Hilb}_{h}\left(X, \mathbb{P}^{n}\right)$. It remains to notice that (again, because of the choice of $p$ ) for any $i \geq p$ the correspondence

$$
\mathcal{J} \mapsto H^{0}\left(X, \mathcal{J} \otimes \mathcal{O}_{\mathbb{P}^{n}}(i)\right)
$$

is a regular map (the Grothendieck embedding) of $H_{i l b}\left(X, \mathbb{P}^{n}\right)$ into a Grassmannian.

For (c), it suffices to prove that $\phi \psi=\mathrm{Id}$. We do this at the level of $\mathbb{K}$-points; the proof for $S$-points, where $S$ is any scheme, is similar. So we start with a graded ideal $I \subset A_{[p, q]}$ with $\operatorname{dim}\left(I_{i}\right)=k_{i}$. For any $\nu=0,1, \ldots, p-1$ consider the graded $B$-module

$$
I^{\nu}=\bigoplus_{i \equiv \nu \bmod p} I_{i}
$$


By Theorem 1.3.2 applied to the $B$-module $\bigoplus_{i \equiv \nu \bmod p} A_{i}$, we associate to $I^{\nu}$ a coherent subsheaf $\left.\mathcal{J}^{\nu} \subset \mathcal{O}_{\mathbb{P} n}(\nu)\right|_{X}$. Our statement would follow from the identification

$$
\mathcal{J}^{\nu}=\mathcal{J} \otimes \mathcal{O}_{\mathbb{P}^{n}}(\nu)
$$

which we now prove. Fix some $\nu=0,1, \ldots, p-1$ and consider another Veronese subalgebra

$$
C=\bigoplus_{j} A_{j(p+\nu)} \subset A, \quad C_{j}=A_{j(p+\nu)},
$$

and the corresponding projective embedding $X \subset \mathbb{P}^{M}=\mathbb{P}\left(C_{1}^{*}\right)$. Associate to $I$ the graded $C$-module

$$
K=\bigoplus_{j} I_{j(p+\nu)}
$$

where the summation is over such $j$ that $j(p+\nu) \leq q$. As before, $K$ gives rise to a sheaf of ideals $\mathcal{K} \subset \mathcal{O}_{X}$ whose Hilbert polynomial with respect to the embedding $X \subset \mathbb{P}^{M}$ is equal to $k^{\prime \prime}(t)=k((p+\nu) t)$ and thus its Hilbert polynomial with respect to $X \subset \mathbb{P}^{n}$ is equal to $k$. Thus we have three $\mathbb{K}$-points of $\operatorname{Hilb}_{h}\left(X, \mathbb{P}^{n}\right)$ represented by $\mathcal{J}, \mathcal{J}^{\nu} \otimes \mathcal{O}_{\mathbb{P}^{n}}(-\nu)$ and $\mathcal{K}$. We claim that they all coincide. For this, we consider their images under the Grothendieck embedding into the Grassmannian of subspaces in $H^{0}\left(X, \mathcal{O}_{\mathbb{P}^{n}}(p(p+\nu))\right)$ of dimension $k_{p(p+\nu)}$. Each of these images is equal to the subspace $I_{p(p+\nu)}$. Since the Grothendieck embedding is indeed an embedding, we find that the sheaves of ideals coincide, in particular, that $\mathcal{J}=$ $\mathcal{J}^{\nu} \otimes \mathcal{O}_{\mathbb{P}^{n}}(-\nu)$. This proves (1.3.7) and hence Theorem 1.3.1 is proved.

(1.4) Relative version. Let $S$ be a scheme of finite type and let $\mathcal{A}$ be a commutative $\mathcal{O}_{S}$-algebra which, as an $\mathcal{O}_{S}$-module, is locally free of finite rank. We have then, for each $k \geq 0$, the relative scheme of ideals $J(k, \mathcal{A} / S)$ which is a closed $S$-subscheme in the relative Grassmannian $G(k, \mathcal{A} / S)$ such that for any $S$-scheme $a: Y \rightarrow S$ the set $\operatorname{Hom}_{S}(Y, J(k, \mathcal{A} / S))$ is identified with the set of sheaves of ideals $\mathcal{I} \subset q^{*} \mathcal{A}$ on $Y$ which, as $\mathcal{O}_{Y}$-modules, are locally free direct summands of rank $k$. Similarly for the case of a graded $\mathcal{O}_{S}$-algebra $\mathcal{A}=\bigoplus_{i=a}^{b} \mathcal{A}_{i}$ and a sequence $k=\left(k_{i}\right)_{i=a}^{b}$ of nonnegative integers.

Next, if $p: X \rightarrow S$ is a flat projective morphism and $\mathcal{O}(1)$ is a fixed relative very ample sheaf, then for each $h \in \mathbb{Q}[t]$ we have the relative Hilbert scheme $\operatorname{Hilb}_{h}(X / S) \rightarrow S$.

If we define $\mathcal{A}_{i}=p_{*} \mathcal{O}(i)$, then for $i \gg 0$ each $\mathcal{A}_{i}$ is locally free of finite rank on $S$, so for $0 \ll p \leq q$ the relative graded ideal space $J\left(k, \mathcal{A}_{[p, q]} / S\right)$ is defined. The arguments of this section relativize immediately to give the following fact.

(1.4.1) Theorem. If $k_{i}=h^{\mathcal{O}_{X}}(i)-h(i)$, then for $0 \ll p \ll q$ the natural morphism $\operatorname{Hilb}_{h}(X / S) \rightarrow J\left(k, \mathcal{A}_{[p, q]} / S\right)$ is an isomorphism of $S$-schemes.

\section{RevieW of COTANGENT COMPLEXES}

In this section we review, for future reference, the formalism of relative cotangent complexes. Our approach, equivalent to that of Illusie [I], is based on dg-resolutions, rather than simplicial resolutions. From now on we assume that our base field $\mathbb{K}$ has characteristic 0 . 
(2.1) Generalities. Let $f: X \rightarrow Y$ be a morphism of quasi-projective dg-schemes. By Theorem 2.7.6 of [CK], we can factor $f$ into a composition $X \stackrel{i}{\hookrightarrow} \widetilde{X} \stackrel{\widetilde{f}}{\rightarrow} Y$, where $i$ is a quasi-isomorphic closed embedding and $\tilde{f}$ is a smooth morphism. The relative (derived) cotangent complex $L \Omega_{X / Y}^{1 \bullet}$ is, by definition, the dg-sheaf $\Omega_{\widetilde{X} / Y}^{1 \bullet}$ of relative 1 -forms for $\widetilde{X} \rightarrow Y$. This is a dg-sheaf (in fact, a dg-bundle, in the sense of [CK], Def. 2.3.2) on $\widetilde{X}$. It is well defined up to quasi-isomorphism in the sense explained in Proposition 2.7.7 of [CK]. We will denote

$$
\mathbb{L}_{X / Y}^{\bullet}=i^{*} \Omega_{\widetilde{X} / Y}^{1 \bullet}
$$

This is a quasi-free dg-sheaf on $X$, well defined up to quasi-isomorphism. Note also that we have a natural projection (restriction map) $L \Omega_{X / Y}^{1 \bullet} \rightarrow i_{*} \mathbb{L}_{X / Y}^{\bullet}$ of dgsheaves on $\tilde{X}$. This is a quasi-isomorphism, as one sees from the Eilenberg-Moore spectral sequence ([CK], Prop. 2.4.1).

(2.1.1) Examples. (a) Note, in particular, the case when $X, Y$ are usual schemes (trivial dg-structure). In this case the formalism of relative cotangent complexes was developed by Illusie [I]. It produces a simplicial sheaf $L_{\bullet}^{\text {Ill }}(X / Y)$ of $\mathcal{O}_{X}$-modules by using a simplicial resolution of $\mathcal{O}_{X}$ as an $f^{-1} \mathcal{O}_{Y}$-algebra. It is not hard to see that the $\mathbb{Z}_{-}$-graded cochain complex associated to $L_{\bullet}^{\text {Ill }}(X / Y)$ is quasi-isomorphic to $\mathbb{L}_{X / Y}$. More precisely, we need to use the equivalence between simplicial commutative algebras and $\mathbb{Z}_{-}$-graded dg-algebras over a field of characteristic 0 .

(b) If $f: X \rightarrow Y$ is a smooth map of usual quasi-projective schemes, then we can take $\widetilde{X}=X$, and $L \Omega_{X / Y}^{1 \bullet}=\mathbb{L}_{X / Y}^{\bullet}=\Omega_{X / Y}^{1}$ is the usual sheaf of relative 1-forms.

(c) Let $Y$ be a smooth quasi-projective variety and let $f: X \hookrightarrow Y$ be the embedding of a local complete intersection. If $\mathcal{I} \subset \mathcal{O}_{Y}$ is the sheaf of ideals of $X$, then $\mathcal{I} / \mathcal{I}^{2}$ is a locally free sheaf on $X$, called the conormal bundle of $X$ in $Y$ and denoted $\mathcal{N}_{X / Y}^{*}$. In this case it is easy to see by using Koszul resolutions that $\mathbb{L}_{X / Y}$ is quasi-isomorphic to $\mathcal{N}_{X / Y}^{*}$ placed in degree $(-1)$.

(d) If $\phi: A \rightarrow B$ is a morphism of finitely generated commutative algebras, we have a morphism of affine $\operatorname{schemes} \phi^{*}: \operatorname{Spec}(B) \rightarrow \operatorname{Spec}(A)$. We will use the notation $L \Omega_{B / A}^{1 \bullet}$ for the relative cotangent complex of this morphism. By definition, $L \Omega_{B / A}^{1 \bullet}=\Omega_{\widetilde{B} / A}^{1 \bullet}$, where $\widetilde{B} \rightarrow B$ is an $A$-algebra resolution of $B$ such that $\operatorname{Spec}(\widetilde{B}) \rightarrow$ $\operatorname{Spec}(A)$ is smooth (in particular, $\widetilde{B}$ is assumed to have finitely many generators in each degree). Accordingly, we write $\mathbb{L}_{B / A}^{\bullet}=\Omega_{\widetilde{B} / A}^{1 \bullet} \otimes_{\widetilde{B}} B$. This is a complex of $B$-modules well defined up to quasi-isomorphism.

In fact, in the affine case we can use more general types of resolutions, not necessarily finitely generated. More precisely, an $A$-dg-algebra $F$ is called quasifree, if $F^{\sharp}$ is free over $A^{\sharp}$ as a graded commutative algebra.

(2.1.2) Proposition. If, in the situation of Example 2.1.1(d), $F \rightarrow B$ is any resolution of $B$ by a quasi-free $A$-dg-algebra, then $L \Omega^{1 \bullet}(B / A)$ is quasi-isomorphic to $\Omega^{1 \bullet}(F / A)$, the dg-module of relative 1 -forms, and hence $\mathbb{L}_{B / A}=\Omega^{1 \bullet}(F / A) \otimes_{F} B$.

Proof. The simplicial approach of Illusie, see Example 2.1.1(a), works without regard to finiteness conditions. The simplicial module $L_{\bullet}^{\mathrm{IIl}}(B / A)$ is obtained by forming a simplicial free resolution, namely the bar-resolution, of $B$ as an $A$-algebra. In 
order to relate this to our definition, we should mix the two approaches by defining $L_{\bullet}^{\text {Ill }}$ for dg-algebras (which is done in an obvious way) and then prove that for a quasi-free $A$-dg-algebra $F$ the dg-module obtained from a simplicial dg-module $L_{\bullet}^{\text {Ill }}(F / A)$ is quasi-isomorphic to $\mathbb{L}_{F / A}^{\bullet}$. A spectral sequence argument reduces this statement to the case when $F$ has trivial differential, so is just a free graded commutative algebra over $A$. In this case the proof is similar to that for a free ungraded algebra which is well known [I].

(2.2) The graded version. In this paper we will allow the dg-algebras (in particular, commutative algebras with trivial dg-structure) to have an extra grading indicated in the subscript: $A=\bigoplus_{i} A_{i}$ (cf. Section 1) and not contributing to the Koszul sign rule. We will call the upper degree cohomological and lower degree projective.

If $A=\bigoplus A_{i}$ is a finitely generated graded commutative algebra (with trivial cohomological grading), then we denote, as usual, by $\operatorname{Proj}(A)$ the projective spectrum of $A$ and by $\mathcal{O}(1)$ the standard invertible sheaf on it. For a graded $A$-module $M=\bigoplus M_{i}$ we denote by $\operatorname{Sh}(M)$ the quasi-coherent sheaf on $\operatorname{Proj}(A)$ obtained from $M$ by localization. For a quasi-coherent sheaf $\mathcal{F}$ on $\operatorname{Proj}(A)$ we denote by $\operatorname{Mod}(\mathcal{F})$ the graded $A$-module $\bigoplus H^{0}(\operatorname{Proj}(A), \mathcal{F}(i))$. It is well known that $\operatorname{Sh}(\operatorname{Mod}(\mathcal{F}))=\mathcal{F}$ while in general there is only a map $M \rightarrow \operatorname{Mod}(\operatorname{Sh}(M))$ which, for $M$ finitely generated, is an isomorphism in sufficiently high degrees.

We now extend this to dg-algebras. Let $A$ be a bigraded dg-algebra as above with finitely many generators in each cohomological degree. Then we have a dg-scheme $\operatorname{Proj}(A)=\left(\operatorname{Proj}\left(A_{\bullet}^{0}\right), \mathcal{O}^{\bullet}\right)$, where $\mathcal{O}^{i}$ is the sheaf $\operatorname{Sh}\left(A_{\bullet}^{i}\right)$, where $A_{\bullet}^{i}$ is regarded as an $A_{\bullet}^{0}$-module. Recall $\mathrm{CK}$ that a dg-scheme $X$ is called projective if $X^{0}$ is projective and each $\mathcal{O}_{X}^{i}$ is coherent. From the above we easily get the following.

(2.2.1) Proposition. Any projective dg-scheme $X$ can be obtained as $\operatorname{Proj}(A)$, if we take $A^{i}=\operatorname{Mod}\left(\mathcal{O}_{X}^{i}\right)$.

For $X=\operatorname{Proj}(A)$ and a bigraded $A$-module $M$ we have a quasi-coherent dgsheaf $\operatorname{Sh}(M)$ on $X$; explicitly, we take $\operatorname{Sh}(M)^{i}=\operatorname{Sh}\left(M_{\bullet}^{i}\right)$. Conversely, for a quasicoherent dg-sheaf $\mathcal{F}^{\bullet}$ on $X$ we form a bigraded $A$-module $\operatorname{Mod}\left(\mathcal{F}^{\bullet}\right)$ defined by $\operatorname{Mod}\left(\mathcal{F}^{\bullet}\right)_{\bullet}^{i}=\operatorname{Mod}\left(\mathcal{F}^{i}\right)$. It is clear that $\operatorname{Sh}(\operatorname{Mod}(\mathcal{F}))=\mathcal{F}$.

Let $A=\bigoplus A_{i}$ and $B=\bigoplus B_{i}$ be two bigraded commutative dg-algebras and let $\phi: A \rightarrow B$ be a morphism of dg-algebras preserving the grading. In this case the relative cotangent complex $L \Omega_{B / A}^{1 \bullet}$ also acquires extra grading, induced by those in $A, B$. According to our convention, we denote the $i$ th graded component of this complex by $L \Omega_{B / A, i}^{1 \bullet}$.

Denote $X=\operatorname{Proj}(B)$ and $Y=\operatorname{Proj}(A)$, and suppose that we have a morphism $f: X \rightarrow Y$ induced by a morphism $\phi: A \rightarrow B$ of bigraded dg-algebras. We would like to compare $L \Omega_{A / B}^{1} \bullet$ with $L \Omega_{X / Y}^{1}$. To construct the former, it is sufficient to form a free bigraded resolution $\widetilde{B}$ of $B$ as an $A$-algebra and we can assume that $\widetilde{B}$ has finitely many generators in each cohomological degree. Then, we can think of $L \Omega_{A / B}^{1 \bullet}=\Omega_{\widetilde{B} / A}^{1 \bullet}$ as a bigraded $\widetilde{B}$-module. Geometrically, the dg-scheme $\widetilde{X}=\operatorname{Proj}(\widetilde{B})$ is a kind of projective space over $Y=\operatorname{Proj}(A)$; in particular, we get a factorization of $f$ as $X=\operatorname{Proj}(B) \subset \widetilde{X} \stackrel{\widetilde{f}}{\rightarrow} Y$, and this factorization can be used to construct the relative tangent complex $L \Omega_{X / Y}^{1 \bullet}=\Omega{\underset{X}{\mathscr{X} / Y}}^{\bullet}$ and its restriction $\mathbb{L}_{X / Y}^{\bullet}$. 
(2.2.2) Theorem. We have the identification of coherent dg-sheaves on $\widetilde{X}$ :

$$
\operatorname{Sh}\left(L \Omega_{B / A}^{1 \bullet}\right)=L \Omega_{X / Y}^{1 \bullet}
$$

Proof. First, assume that $X$ and $Y$ are ordinary (not dg) smooth varieties and $f$ is a closed embedding. In this case $\mathbb{L}_{X / Y}$ is equal to $\mathcal{N}_{X / Y}^{*}[1]=\mathcal{I} / \mathcal{I}^{2}[1]$, where $\mathcal{I} \subset \mathcal{O}_{Y}$ is the sheaf of ideals of $X$. Let us denote by 0 the "origin" in $\operatorname{Spec}(A)$ and $\operatorname{Spec}(B)$, i.e., the point corresponding to the ideal of elements of positive degree. Then, up to modules supported at 0 , we have that $L \Omega_{B / A}^{1 \bullet}$ is quasi-isomorphic to $I / I^{2}[1]$, where $I=\operatorname{Ker}(\phi)$. So our statement in the particular case we consider follows from the next easy lemma.

(2.2.3) Lemma. We have $\operatorname{Sh}\left(I^{\nu}\right)=\mathcal{I}^{\nu}$, for each $\nu \geq 0$.

Now consider the general case and form the fiber product $\widetilde{X}^{2}:=\widetilde{X} \times_{Y} \widetilde{X}$, with diagonal $\Delta: \widetilde{X} \longrightarrow \widetilde{X}^{2}$. Let $\mathcal{I} \subset \mathcal{O}_{\widetilde{X}^{2}}$ be the dg-ideal of the diagonal. Then $L \Omega_{X / Y}^{1 \bullet}=\mathcal{I} / \mathcal{I}^{2}$. Similarly, consider the dg-algebra $\widetilde{B}^{2}:=\widetilde{B} \otimes_{A} \widetilde{B}$ and let $I$ be the kernel of the multiplication homomorphism $\widetilde{B}^{2} \longrightarrow \widetilde{B}$. Then $L \Omega_{B / A}^{1 \bullet}$ is quasiisomorphic to $I / I^{2}$, up to dg-modules supported at the origin. We conclude the argument by using an obvious dg-version of Lemma 2.2.3.

(2.3) Cotangent complex and Harrison complex. Recall an explicit construction of the absolute cotangent complex in the affine case via Harrison chains [Lo].

Let $A$ be a commutative $\mathbb{K}$-algebra. We denote by $\operatorname{FCoLie}(A[1])$ the free graded Lie coalgebra cogenerated by the graded vector space $A[1]$. Thus FCoLie $(A[1])=$ $A \oplus S^{2}(A) \oplus \ldots$... Let $d$ be the unique differential on FCoLie $(A[1])$ which is compatible with the Lie coalgebra structure and on the space of cogenerators is given by the multiplication $S^{2}(A) \rightarrow A$. The resulting complex (dg-Lie coalgebra)

$$
(\operatorname{FCoLie}(A[1]), d)=\operatorname{Harr} \bullet(A, \mathbb{K})
$$

is known as the Harrison chain complex of $A$ with coefficients in $\mathbb{K}$. More generally, if $M$ is an $A$-module, then the graded vector space

$$
\operatorname{Harr} \bullet(A, M)=\operatorname{FCoLie}(A[1]) \otimes_{\mathbb{K}} M
$$

has a natural differential making it into a dg-comodule over $\operatorname{Harr} .(A, \mathbb{K})$. It is known as the Harrison chain complex with coefficients in $M$. Dually, we have the Harrison cochain complex

$$
\begin{gathered}
\operatorname{Harr}^{\bullet}(A, M)=\operatorname{Hom}_{\mathbb{K}}(\mathrm{FCoLie}(A[1]), M) \\
=\left\{\operatorname{Hom}_{\mathbb{K}}(A, M) \stackrel{\delta}{\longrightarrow} \operatorname{Hom}_{\mathbb{K}}\left(S^{2}(A), M\right) \longrightarrow \ldots\right\}
\end{gathered}
$$

with the first differential acting by the standard formula

$$
(\delta f)(a \cdot b)=f(a b)-a f(b)-b f(a) .
$$

This means that

$$
H^{1} \operatorname{Harr}^{\bullet}(A, M)=\operatorname{Der}(A, M), \quad H^{-1} \operatorname{Harr}(A, M)=\Omega_{A / k}^{1} \otimes M .
$$

Now, the standard property of the Harrison complex is as follows.

(2.3.1) Theorem. The complex Harr. $(A, A)$ is quasi-isomorphic to $\mathbb{L}_{A / k}^{\bullet}$. 
Proof. This follows from Proposition 2.1.2, applied to a particular quasi-free resolution of $A$, namely the commutative bar-resolution

$$
S \mathcal{L}(A):=(\operatorname{FCoLie}(A[1])[-1]) \stackrel{\sim}{\longrightarrow} A .
$$

\section{A Finite-Dimensional MODEL}

Let $A$ be a finite-dimensional commutative algebra over $\mathbb{K}$, possibly without unit. For $k>0$, let $J(k, A)$ be the scheme of $k$-dimensional ideals of $A$ (see (1.2)). In this section we construct a dg-manifold $R J(k, A)$, with $\pi_{0}(R J(k, A))=J(k, A)$ and

$$
H^{i} T_{[I]}^{\bullet} R J(k, A)=\operatorname{Ext}_{A / I}^{i+1}\left(\mathbb{L}_{(A / I) / A)}, A / I\right),
$$

for any $\mathbb{K}$-point $I \in J(k, A)$. As in [CK], this is achieved by representing $J(k, A)$ in terms of two abstract constructions, which we now explain.

\section{(3.1) Two constructions.}

(3.1.1). Let $W$ be a finite-dimensional vector space over $\mathbb{K}$. We consider the subscheme $C A(W)$ of $\operatorname{Hom}\left(S^{2} W, W\right)$ formed by all commutative, associative multiplications on $W$. It is clear that for any $\mathbb{K}$-point $[\mu] \in C A(W)$, represented by $\mu: S^{2} W \longrightarrow W$, we have that

$$
T_{[\mu]} C A(W)=Z_{\text {Harr }}^{2}(W, W) .
$$

Similarly, if $W$ is a vector bundle over a scheme $S$, we get the relative space of algebra structures $C A(W) \longrightarrow S$.

(3.1.2). Let $S$ be a scheme and let $A$ and $B$ be vector bundles over $S$ which are made into commutative $\mathcal{O}_{S}$-algebras. Let $f: A \longrightarrow B$ be a morphism of $\mathcal{O}_{S}$-modules. The homomorphicity locus $M_{f}$ is informally the locus of points $s \in S$ such that the morphism of fibers $f_{s}: A_{s} \longrightarrow B_{s}$ is an algebra homomorphism. More precisely, $M_{f}$ is the following fiber product:

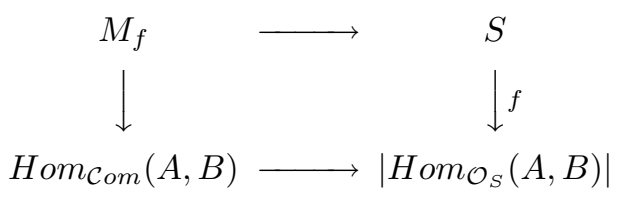

Here $|E|$ means the total space of a vector bundle $E$ and $H_{o m} m_{\mathcal{C} o m}$ is the subscheme formed by homomorphisms of commutative algebras.

We apply these constructions to the following situation. We take $A$ to be a finitedimensional algebra, $G(k, A)$ the Grassmannian of $k$-dimensional subspaces, and $\tilde{V}$ the tautological rank $k$-bundle over $G(k, A)$, whose fiber over a point $[V] \in G(k, A)$ corresponding to a subspace $V \subset A$ is $V$. Thus $\tilde{V}$ is a subbundle in $A$ (the trivial bundle with fiber $A$ ). Let $A / \tilde{V}$ be the quotient bundle. Consider the relative scheme of algebra structures on the fibers of this bundle:

$$
C A(A / \tilde{V}) \stackrel{q}{\longrightarrow} G(k, A) .
$$

On this scheme we have a canonical morphism of vector bundles

$$
f: A \rightarrow q^{*}(A / \tilde{V}) \text {. }
$$

These vector bundles are in fact commutative $\mathcal{O}_{C A(A / \tilde{V})}$-algebras.

(3.1.3) Proposition. The homomorphicity locus $M_{f}$ is isomorphic to $J(k, A)$. 
Proof. Almost a tautology. Namely, a $k$-linear subspace $I \subset A$ is an ideal if and only if $A / I$ is made into a commutative algebra so that the natural projection $A \rightarrow A / I$ is an algebra homomorphism.

Now we construct the derived variety of ideals by developing the derived versions of each of the two constructions above.

(3.2) Derived space of algebra structures. Our approach is similar to that of C. Rezk [Re] and parallels our earlier construction [CK] for structures of a module, not an algebra.

First, we generalize the concept of the space of algebra structures. Let $\mathcal{P}=$ $\{\mathcal{P}(n), n \geq 0\}$ be a $\mathbb{K}$-linear operad $\mathrm{GK}$. Thus, by definition, each $\mathcal{P}(n)$ is acted upon by the symmetric group $S_{n}$ and $\mathcal{P}$ is equipped with composition maps

$$
\mathcal{P}(n) \otimes \mathcal{P}\left(a_{1}\right) \otimes \ldots \otimes \mathcal{P}\left(a_{n}\right) \rightarrow \mathcal{P}\left(a_{1}+\ldots+a_{n}\right)
$$

satisfying the axioms of May. For example, if $W$ is a vector space, then we have the endomorphism operad $\mathcal{E}_{W}$ with $\mathcal{E}_{W}(n)=\operatorname{Hom}\left(W^{\otimes n}, W\right)$ and the maps (3.2.1) given by composition of multilinear maps. A $\mathcal{P}$-algebra structure on $W$ is a morphism of operads $\mathcal{P} \rightarrow \mathcal{E}_{W}$. For example, the case of ordinary commutative algebras (possibly without unit) corresponds to the case when $\mathcal{P}$ is the commutative operad $\mathcal{C}$ om with $\mathcal{C}$ om $(n)=\mathbb{K}$ with trivial $S_{n}$-action, for any $n \geq 1$.

Now let $W$ be a finite-dimensional vector space and let $\mathcal{P}$ be any operad. In this case we have a scheme $\mathcal{P} \operatorname{Alg}(W)$ parametrizing $\mathcal{P}$-algebra structures on $W$. It is realized as a closed subscheme in the (possibly infinite-dimensional) affine space:

$$
\mathcal{P} A l g(W) \subset \prod_{n}\left|\operatorname{Hom}_{S_{n}}\left(\mathcal{P}(n), \mathcal{E}_{W}(n)\right)\right|,
$$

given by the equations of "compatibility with operad structures". Note that even when $\mathcal{P}(n)$ is infinite dimensional, $\left|\operatorname{Hom}_{S_{n}}\left(\mathcal{P}(n), \mathcal{E}_{W}(n)\right)\right|$ still makes sense as a scheme, the spectrum of a polynomial ring in infinitely many variables.

There is a case when the scheme of $\mathcal{P}$-algebra structures can be found explicitly. This is the case of free operads, which we now recall. Let $E$ be an $S$-module GK], i.e., a collection $E=\{E(n), n \geq 0\}$ of vector spaces together with an $S_{n}$-action on $E(n)$ given for each $n$. To $E$, there corresponds the free operad $F_{E}$ characterized by the condition that for any other operad $\mathcal{P}$

$$
\operatorname{Hom}_{\text {Operads }}\left(F_{E}, \mathcal{P}\right)=\operatorname{Hom}_{S \text {-modules }}(E, \mathcal{P}) \text {. }
$$

It follows that

$$
F_{E} \operatorname{Alg}(W)=\prod_{n}\left|\operatorname{Hom}_{S_{n}}\left(E(n), \operatorname{Hom}\left(W^{\otimes n}, W\right)\right)\right|
$$

is a (possibly infinite-dimensional) affine space.

We now extend the above formalism to $\mathbb{Z}_{-}$-graded dg-operads, i.e., operads in the symmetric monoidal category of $\mathbb{Z}_{-}$-graded cochain complexes. For such an operad $\mathcal{P}$, we will denote by $\mathcal{P}_{\sharp}$ the graded operad which is the same as $\mathcal{P}$ but with differential forgotten. We say that a dg-operad $\mathcal{F}$ is quasi-free if $\mathcal{F}_{\sharp}$ is free in the above sense.

(3.2.5) Proposition. Let $W$ be a finite-dimensional $\mathbb{K}$-vector space and let $\mathcal{P}$ be any $\mathbb{Z}_{-}$-graded dg-operad. Then there exists an affine dg-scheme $\mathcal{P} A l g(W)$ such that for any commutative dg-algebra $A$ morphisms $\operatorname{Spec}(A) \rightarrow \mathcal{P} A l g(W)$ are in bijection with $A \otimes_{\mathbb{K}} \mathcal{P}$-algebra structures in the $A$-module $A \otimes_{\mathbb{K}} W$. 
Proof. First, consider the case when $\mathcal{P}$ has trivial differential (but possibly nontrivial grading). Then, we have a version of the embedding (3.2.2) with the RHS being the graded affine space, namely the spectrum of the $\mathbb{Z}_{-}$-graded commutative algebra

$$
\bigotimes_{n} S^{\bullet}\left(\left(\mathcal{P}(n) \otimes \mathcal{E}_{W}(n)^{*}\right)^{S_{n}}\right) .
$$

The coordinate ring of the LHS, i.e., of the sought-for scheme $\mathcal{P} A l g(W)$, is obtained by quotienting (3.2.6) by the graded ideal formed by the equations expressing compatibility with operad structures. The graded scheme $\mathcal{P} \operatorname{Alg}(W)$ is, by construction, functorial in $\mathcal{P}$ and $W$.

Next, suppose that the differential in $\mathcal{P}$ is nontrivial and consider the graded scheme $\mathcal{P}_{\sharp} A l g(W)$. The differential $d$ in $\mathcal{P}$ can be regarded as an odd infinitesimal automorphism of $\mathcal{P}_{\sharp}$; see, e.g., [Ka], Prop. 1.1.1. So the graded scheme $\mathcal{P}_{\sharp} A l g(W)$ inherits this action by naturality, i.e., we get a dg-scheme which we denote $\mathcal{P} \operatorname{Alg}(W)$. The determination of the functor represented by $\mathcal{P} \operatorname{Alg}(W)$ is straightforward.

(3.2.7) Proposition. (a) Any $\mathbb{Z}_{-}$-graded dg-operad $\mathcal{P}$ possesses a quasi-free resolution $\mathcal{F}$. Moreover, if each graded piece of each $\mathcal{P}(n)$ is finite dimensional, we can find $\mathcal{F}$ so that $\mathcal{F}_{\sharp}=F_{E}$ and each graded component of each $E(n)$ is finite dimensional.

(b) If $q: \mathcal{F}_{1} \rightarrow \mathcal{F}_{2}$ is a quasi-isomorphism of quasi-free dg-operads, then for any finite-dimensional vector space $W$ the induced morphism of dg-schemes $q^{*}$ : $\mathcal{F}_{2} \operatorname{Alg}(W) \rightarrow \mathcal{F}_{1} A l g(W)$ is a quasi-isomorphism.

Part (a) is clear, and part (b) will be proved in (3.4). Assuming the proposition, we can give the following definition.

(3.2.8) Definition. Let $\mathcal{P}$ be any $\mathbb{Z}_{-}$-graded dg-operad and let $W$ be a finitedimensional vector space. Then the derived space of $\mathcal{P}$-actions on $W$ is defined to be

$$
R \mathcal{P} A l g(W)=\mathcal{F} \operatorname{Alg}(W),
$$

where $\mathcal{F} \rightarrow \mathcal{P}$ is any quasi-free resolution.

(3.3) The bar-resolution for operads. We now describe a particular functorial quasi-free resolution of any dg-operad [GK].

First of all, if $E^{\bullet}=\left\{E^{\bullet}(n)\right\}$ is any graded $S$-module, its suspension is the graded $S$-module $\Sigma E^{\bullet}$ defined by

$$
\left(\Sigma E^{\bullet}\right)(n)=E^{\bullet}(n)[1-n] \otimes \operatorname{sgn}_{n},
$$

where $\operatorname{sgn}_{n}$ is the sign representation of $S_{n}$; see GK]. The inverse functor will be denoted by $\Sigma^{-1}$. Now let $\mathcal{P}$ be any $\mathbb{Z}_{-}$-graded dg-operad.

(3.3.2) Proposition. (a) For any $\mathbb{Z}_{-}$-graded cooperad $\mathcal{C}$ the free operad $F_{\Sigma^{-1} \mathcal{C}}$ has a natural differential $d^{\prime}$ (making it into a dg-operad) which on the cogenerators is induced by the cocomposition in $\mathcal{C}$.

(b) If $\mathcal{C}$ is a $\mathbb{Z}_{-}$-graded dg-cooperad with differential $d_{\mathcal{C}}$, then the induced differential $d^{\prime \prime}$ on $F_{\Sigma^{-1} \mathcal{C}_{\sharp}}$ commutes with $d^{\prime}$ and the total differential $d=d^{\prime}+d^{\prime \prime}$ makes $F_{\Sigma^{-1} \mathcal{C}}$ into a dg-operad which we denote $\operatorname{Cobar}(\mathcal{C})$. 
This construction is known as the cobar-construction of the cooperad $\mathcal{C}$; see GK] GJ]. Similarly, we have the bar-construction for operads.

(3.3.3) Proposition. (a) For any $\mathbb{Z}_{-}$-graded operad $\mathcal{P}$ the free cooperad $\operatorname{Bar}(\mathcal{P})$ generated by $\Sigma \mathcal{P}$ has a natural differential d' (making it into a dg-cooperad) which on the space of generators is induced by the composition in $\mathcal{P}$.

(b) If $\mathcal{P}$ is a $\mathbb{Z}_{-}$-graded dg-operad with differential $d_{\mathcal{P}}$, then the induced differential $d^{\prime \prime}$ on $\operatorname{Bar}\left(\mathcal{P}_{\sharp}\right)$ commutes with $d^{\prime}$ and the total differential $d=d^{\prime}+d^{\prime \prime}$ makes it into a dg-cooperad which we denote $\operatorname{Bar}(\mathcal{P})$.

By construction, Bar and Cobar are functors from dg-operads to dg-cooperads and back, and it is easy to see that these functors take quasi-isomorphisms to quasi-isomorphisms.

We now define the bar-resolution of a dg-operad $\mathcal{P}$ to be

$$
\mathcal{B}(\mathcal{P})=\operatorname{Cobar}(\operatorname{Bar}(\mathcal{P})) \text {. }
$$

Thus, $\mathcal{B}(\mathcal{P})$ is quasi-free and functorial in $\mathcal{P}$.

(3.3.5) Proposition. There is a natural quasi-isomorphism $\alpha: \mathcal{B}(\mathcal{P}) \rightarrow \mathcal{P}$.

For a vector space $W$ this particular quasi-free resolution gives a particular model for the derived space of $\mathcal{P}$-algebra structures which we denote

$$
\widetilde{R} \mathcal{P} A l g(W)=\mathcal{B}(\mathcal{P}) \operatorname{Alg}(W) .
$$

A $\mathcal{B}(\mathcal{P})$-algebra $W$ is sometimes called a homotopy $\mathcal{P}$-algebra. More precisely, we have maps $\mathcal{P}(n) \otimes W^{\otimes n} \rightarrow W$ which satisfy the axioms of an operad action only up to higher homotopies.

In the same way as for Proposition 3.5.3 of [CK], we prove the following.

(3.3.7) Proposition. For any $\mathbb{Z}_{-}$-graded $d g$-operad $\mathcal{P}$ we have natural convergent spectral sequences

$$
\begin{aligned}
& E_{1}=H^{\bullet} \mathbb{K}\left[\widetilde{R} \mathcal{P}_{\sharp} A l g(W)\right] \quad \Rightarrow \quad H^{\bullet} \mathbb{K}[\widetilde{R} \mathcal{P} \operatorname{Alg}(W)] ; \\
& E_{2}=\mathbb{K}\left[\widetilde{R} H^{\bullet}(\mathcal{P}) A l g(W)\right] \quad \Rightarrow \quad H^{\bullet} \mathbb{K}[\widetilde{R} \mathcal{P} \operatorname{Alg}(W)] .
\end{aligned}
$$

(3.4) M-homotopies and the proof of Proposition 3.2.7. Let $f: \mathcal{P} \rightarrow \mathcal{Q}$ be a morphism of dg-operads. Then $f$ makes $\mathcal{Q}$ into a bimodule over $\mathcal{P}$ in the sense of $[\mathrm{Mar}$. We can, therefore, speak about derivations $D: \mathcal{P} \rightarrow \mathcal{Q}$ with respect to this bimodule structure which are collections of morphisms of complexes $D_{n}: \mathcal{P}(n) \rightarrow \mathcal{Q}(n)$ such that for any $n, a_{1}, \ldots, a_{n} \in \mathbb{Z}_{+}$and any $p \in \mathcal{P}(n), p_{i} \in \mathcal{P}\left(a_{i}\right)$ we have

$$
\begin{gathered}
D_{a_{1}+\ldots+a_{n}}\left(p\left(p_{1}, \ldots, p_{n}\right)\right)=\left(D_{n}(p)\right)\left(f\left(p_{1}\right), \ldots, f\left(p_{n}\right)\right) \\
+\sum_{i=1}^{n} f(p)\left(f\left(p_{1}\right), \ldots, D_{a_{i}}\left(p_{i}\right), \ldots, f\left(p_{n}\right)\right) .
\end{gathered}
$$

Here $p\left(p_{1}, \ldots, p_{n}\right)$ is the image of $p \otimes p_{1} \otimes \ldots \otimes p_{n}$ in $\mathcal{P}\left(a_{1}+\ldots+a_{n}\right)$ under the composition map in $\mathcal{P}$. More generally, we define derivations of degree $d$ by allowing the $D_{n}$ to be morphisms of complexes of degree $d$ and introducing obvious sign factors.

It is clear that whenever $\left(f_{t}: \mathcal{P} \rightarrow \mathcal{Q}\right)_{t \in \mathbb{K}}$ is a polynomial family of morphisms, then for each $t$ the derivative $f_{t}^{\prime}=\frac{d}{d t} f_{t}$ is a derivation $\mathcal{P} \rightarrow \mathcal{Q}$ with respect to the bimodule structure given by $f_{t}$. 
(3.4.1) Definition. An $M$-homotopy between two morphisms $f, g: \mathcal{P} \rightarrow \mathcal{Q}$ is a pair $\left(f_{t}, s_{t}\right)$, where $\left(f_{t}\right)_{t \in \mathbb{K}}$ is a polynomial family of morphisms of dg-operads such that $f_{0}=f, f_{1}=g$ and $\left(s_{t}\right)$ is a polynomial family of degree $(-1)$ derivations $\mathcal{P} \rightarrow \mathcal{Q}$ (with respect to the bimodule structure given by $f_{t}$ ) such that $f_{t}^{\prime}=\left[d, s_{t}\right]$.

As in the case for algebras $\mathrm{CK}$, we see that two $M$-homotopic morphisms $\mathcal{P} \rightarrow \mathcal{Q}$ induce the same morphism $H^{\bullet}(\mathcal{P}) \rightarrow H^{\bullet}(\mathcal{Q})$.

(3.4.2) Lemma. Let $\mathcal{P}, \mathcal{Q}$ be $\mathbb{Z}_{-}$-graded dg-operads such that $\mathcal{P}$ is quasi-free and each $\mathcal{Q}(n)$ is acyclic in degrees $<0$. Suppose that $f_{1}, f_{2}: \mathcal{P} \rightarrow \mathcal{Q}$ are two morphisms of dg-operads which, for each $n$, induce the same morphisms $H^{0} \mathcal{P}(n) \rightarrow H^{0} \mathcal{Q}(n)$. Then there exists an $M$-homotopy connecting $f_{0}$ and $f_{1}$.

Proof. This is similar to the proof of Proposition 3.6.4 of [CK] which deals with an analogous statement but for associative algebras instead of operads. More precisely, the inductive (in the homological degree) procedure from that proof adapts without difficulty to the case of operads.

(3.4.3) Corollary. Let $\mathcal{R}=F_{E}$ be the free graded operad on a $\mathbb{Z}_{-}$-graded $S$ module $E$ (no differential). Then the quasi-isomorphism $\alpha: \mathcal{B}(\mathcal{R}) \rightarrow \mathcal{R}$ from Proposition 3.3.5 has a natural left inverse $\beta: \mathcal{R} \rightarrow \mathcal{B}(\mathcal{R})$ and the composition $\beta \alpha$ is $M$-homotopic to the identity of $\mathcal{B}(\mathcal{R})$.

Proof. As $\mathcal{R}$ is free, we can define a morphism from $\mathcal{R}$ by prescribing its restriction to the $S$-module of generators. We define $\beta$ on $E$ to be the canonical identification of it with the natural copy of $E$ inside $\mathcal{R} \subset F_{\mathcal{R}} \subset \mathcal{B}(\mathcal{R})$. Now, if $E$ is concentrated in degree 0 (i.e., each $E(n)$ is), then we can apply Lemma 3.4 .2 to $\mathcal{P}=\mathcal{Q}=\mathcal{B}(\mathcal{R})$. If $E$ is not concentrated in degree 0 , then we notice that $\mathcal{B}(\mathcal{P})$ comes from a $\mathbb{Z}_{-} \times \mathbb{Z}_{-}$graded dg-operad with differential of degree $(1,0)$ and then use the same proof as for Lemma 3.4.2, but with induction in the second component of the bidegree.

We now pass to the proof of Proposition 3.2.7. Again, we use the same method as was used for the proof of Proposition 3.3.6 of [CK so we will just outline the main steps.

First, we prove that if $\mathcal{P}=F_{E}$ is free with trivial differential, then $\widetilde{R} \mathcal{P} A l g(W)$ is quasi-isomorphic to $\mathcal{P} A l g(W)$. This is done by using Corollary 3.4.3 to $\mathcal{R}=\mathcal{P}$ and noticing that the identity $\alpha \beta=\operatorname{Id}$ and the $M$-homotopy $\beta \alpha \sim \operatorname{Id}$ between morphisms of operads are inherited in functorial constructions such as passing from an operad $\mathcal{Q}$ to the coordinate algebra $\mathbb{K}[\mathcal{Q} A l g(W)]$.

Then, to finish the argument, we prove that for any quasi-free resolution $p: \mathcal{F} \rightarrow$ $\mathcal{P}$ the dg-algebra $\mathbb{K}[\mathcal{F} A l g(W)]$ is naturally quasi-isomorphic to $\mathbb{K}[\widetilde{R} \mathcal{P} A l g(W)]$ (so is independent, up to quasi-isomorphism, on $\mathcal{F})$. Indeed, by the above $\mathbb{K}\left[\mathcal{F}_{\sharp} A l g(W)\right]$ is quasi-isomorphic to $\mathbb{K}\left[\widetilde{R} \mathcal{F}_{\sharp} A l g(W)\right]$. Then, the first spectral sequence of $3.3 .7 \mathrm{im}-$ plies that $\mathbb{K}[\mathcal{F} A l g(W)] \rightarrow \mathbb{K}[\widetilde{R} \mathcal{F} A l g(W)]$ is a quasi-isomorphism while the second sequence implies that $\mathbb{K}[\widetilde{R} \mathcal{F} A l g(W)] \rightarrow \mathbb{K}[\widetilde{R} \mathcal{P} A l g(W)]$ is a quasi-isomorphism.

(3.5) The small bar-resolution for $\mathcal{P}=\mathcal{C}$ om. We now specialize the above discussion to $\mathcal{P}=\mathcal{C}$ om and write $R C A(W)=R \mathcal{C o m} A l g(W)$. In addition to the bar-resolution $\mathcal{B}(\mathcal{C o m})$ we will use another quasi-free resolution $\Lambda \rightarrow \mathcal{C}$ om which we call the small bar-resolution and which is defined as follows.

Let $\mathcal{L}$ ie be the operad describing Lie algebras. Denote by $\mathcal{L} i e^{*}$ the cooperad formed by the dual spaces $\mathcal{L} i e(n)^{*}$. We set $\Lambda=\operatorname{Cobar}\left(\mathcal{L} i e^{*}\right)$. Because $\mathcal{L} i e^{*}$ is 
quasi-isomorphic to $\operatorname{Bar}(\mathcal{C o m})$ (Koszul duality, see [GK]), we find that $\Lambda$ is quasiisomorphic to $\mathcal{B}(\mathcal{C o m})$; in particular, it is a quasi-free resolution of $\mathcal{C} o m$.

We will denote by $\bar{R} C A(W)$ the particular model for $R C A(W)$ obtained by using the resolution $\Lambda$.

(3.5.1) Proposition. The affine dg-scheme $\bar{R} C A(W)$ is a dg-manifold. In fact, the dg-algebra $\mathbb{K}[\bar{R} C A(W)]_{\sharp}$ is free with finitely many generators in each degree.

Proof. For a vector space $W$ a $\Lambda$-algebra structure on $W$ is the same as a differential $D$ in the free Lie coalgebra on $W[1]$ satisfying $D^{2}=0$ and compatible with the coalgebra structure. So $\Lambda$-algebras are the same as homotopy commutative algebras in the sense of Stasheff [St]. Now, a $D$ as before is defined by the projection of its image on $W[1]$, and this projection can be arbitrary. So $D$ is defined by a collection of linear maps $D_{i}: \mathrm{FCoLie}_{i}(W[1]) \rightarrow W, i \geq 2, \operatorname{deg}\left(D_{i}\right)=2-i$, where $\mathrm{FCoLie}_{i}(W[1])$ is the $i$ th graded component of FCoLie( $\left.W[1]\right)$. Notice that it is finite dimensional. Now, the graded algebra $\mathbb{K}[\bar{R} C A(W)]_{\sharp}$ is, by construction, freely generated by the matrix elements of indeterminate maps $D_{i}$.

(3.5.2) Proposition. Let $W$ be a finite-dimensional vector space. Then:

(a) $\pi_{0} R C A(W)=C A(W)$.

(b) If $\mu: S^{2} W \rightarrow W$ represents a $\mathbb{K}$-point $[\mu] \in C A(W)$, then

$$
H^{i} T_{[\mu]}^{\bullet} R C A(W)= \begin{cases}Z_{\mathrm{Harr}}^{2}(W, W), & i=0 ; \\ H_{\mathrm{Harr}}^{i+2}(W, W), & i>0 .\end{cases}
$$

Proof. Part (a) is obvious from any construction of $R C A$. As to (b), the definition of the Harrison complex uses the free Lie coalgebra on $W[1]$, i.e., exactly the structure involved in the resolution $\Lambda$.

(3.6) The derived homomorphicity locus. Let $A, B$ be two commutative $\mathbb{K}$ algebras and $\operatorname{dim}(B)<\infty$. We then have the scheme of homomorphisms

$$
\operatorname{Hom}_{\mathcal{C o m}}(A, B) \subset\left|\operatorname{Hom}_{\mathbb{K}}(A, B)\right|
$$

whose points are morphisms of commutative algebras $A \rightarrow B$. If $[f]$ is a $\mathbb{K}$-point represented by a homomorphism $f: A \rightarrow B$, then, clearly,

$$
T_{[f]} \operatorname{Hom}_{\mathcal{C o m}}(A, B)=\operatorname{Der}(A, B),
$$

where $B$ is made into an $A$-algebra via $f$.

Now let $A$ be a $\mathbb{Z}_{-}$-graded dg-algebra. Then $\left|\operatorname{Hom}_{\mathbb{K}}(A, B)\right|$ is the $\mathbb{Z}_{-}$-graded dgscheme corresponding to the $\mathbb{Z}_{+}$-graded complex $\operatorname{Hom}_{\mathbb{K}}(A, B)$; see $[\mathrm{CK}, \mathrm{n}$. (2.2.5). The ideal of the graded subscheme $\operatorname{Hom}_{\mathcal{C o m}}\left(A_{\sharp}, B\right) \subset\left|\operatorname{Hom}_{\mathbb{K}}\left(A_{\sharp}, B\right)\right|$ is a differential ideal, so we get the $\operatorname{dg}$-scheme $\operatorname{Hom}_{\mathcal{C o m}}(A, B)$ which parametrizes, in the obvious sense, dg-algebra homomorphisms. We now define the derived scheme of homomorphisms to be

$$
\operatorname{RHom}_{\text {Com }}(A, B)=\operatorname{Hom}_{\mathcal{C o m}}(F, B),
$$

where $F \rightarrow A$ is any quasi-free commutative dg-algebra resolution.

(3.6.3) Lemma. The definition of $\operatorname{RHom}_{\mathcal{C} o m}(A, B)$ is independent, up to quasiisomorphism, on the choice of F. 
Proof. This is done similarly to the proof of Proposition 3.2.7 and of Proposition 3.3.6 of $\mathrm{CK}$, by using the following ingredients: the concept of $M$-homotopies for commutative dg-algebras (parallel to the associative case treated in [CK]) and the canonical quasi-free resolution $\mathcal{L}(A)=S(\operatorname{FCoLie}(A[1])[-1]) \rightarrow A$, which was already used (for ungraded algebras) in (2.3.1).

(3.6.4) Proposition. Let $A, B$ be ungraded commutative algebras, $\operatorname{dim}(B)<\infty$. Then:

(a) $\pi_{0} \operatorname{RHom}_{\mathcal{C o m}}(A, B)=\operatorname{Hom}_{\mathcal{C o m}}(A, B)$.

(b) If $f: A \rightarrow B$ is an algebra homomorphism, then

$$
H^{i} T_{[f]}^{\bullet} \operatorname{RHom}_{\mathcal{C} o m}(A, B)=H_{\text {Harr }}^{i+1}(A, B) .
$$

Note that the Harrison cohomology are the higher derived functors of the space of derivations which, by (3.6.1), is the tangent space to $\operatorname{Hom}_{\mathcal{C} o m}(A, B)$.

Proof. (a) is clear and (b) is obtained by using the resolution $\mathcal{L}(A)$.

We now globalize this construction as follows. Let $S$ be a dg-scheme and let $A, B$ be quasi-coherent sheaves of $\mathcal{O}_{S}$-dg-algebras. We assume that as sheaves of graded $\mathcal{O}_{S \sharp}$-modules, $A_{\sharp}$ and $B_{\sharp}$ are locally free and, moreover, generators of $A_{\sharp}$ are in degrees $\leq 0$ and generators of $B_{\sharp}$ are finite in number and have degree 0 . Then we can apply the construction of the derived homomorphicity locus relative over $S$, getting a dg-scheme $R \mathcal{H}^{\prime} m_{\mathcal{C}} \otimes \mathcal{O}_{S}(A, B)$. It comes equipped with a canonical $S$-map $p$ into the total space $\mid \mathcal{H}$ om $_{\mathcal{O}_{S}}(A, B) \mid$.

Now let $f: A \rightarrow B$ be a morphism of $\mathcal{O}_{S^{-}}$dg-modules. We define the derived homomorphicity locus $R M_{f}$ to be the derived fiber product (in the sense of n. (2.8) of $\mathrm{CK}$ ):

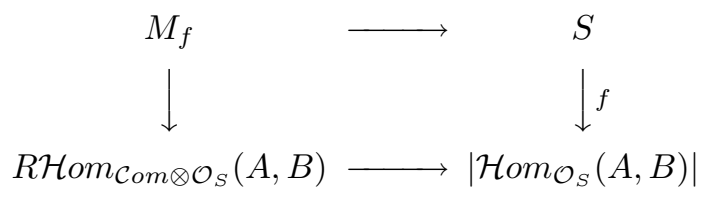

Notice that the construction of the derived homomorphicity locus can be applied to the more general situation when $A$ and $B$ are sheaves of $\mathcal{P} \otimes \mathcal{O}_{S}$-dg-algebras, where $\mathcal{P}$ is any $\mathbb{Z}_{-}$-graded dg-operad (instead of $\mathcal{C}$ om $)$. In particular, we will use this construction for $\mathcal{P}=\Lambda$.

(3.7) The derived ideal scheme. Let $A$ be a finite-dimensional ungraded commutative $\mathbb{K}$-algebra. We now apply the derived versions of the two constructions of (3.1), from which we use the notation. We first consider the relative derived space of algebra structures

$$
R C A(A / \tilde{V}) \stackrel{q}{\longrightarrow} G(k, A) .
$$

As explained, this is done using a quasi-free resolution $\mathcal{F} \rightarrow \mathcal{C}$ om. By construction, $q^{*}(A / \tilde{V})$ is a sheaf of $\mathcal{O}_{R C A(A / \tilde{V})} \otimes \mathcal{F}$-dg-algebras. We have an $\mathcal{O}_{\bar{R} C A(A / \tilde{V})^{-l i n e a r}}$ morphism

$$
f: A \otimes \mathcal{O}_{\bar{R} C A(A / \tilde{V})} \rightarrow q^{*}(A / \tilde{V})
$$

whose source and target are sheaves of $\mathcal{O}_{R C A(A / \tilde{V})} \otimes \mathcal{F}$-dg-algebras. 
(3.7.2) Definition. (a) We define the derived scheme of ideals $R J(k, A)$ to be the derived linearity locus $R M_{f}$.

(b) By $\bar{R} J(k, A)$ we denote the particular model for $R J(k, A)$ obtained by using the resolution $\mathcal{F}=\Lambda$ for the operad $\mathcal{C}$ om and the resolution $\mathcal{L}(A)$ for the algebra $A$.

It is clear from the above that $R J(k, A)$ is independent, up to quasi-isomorphism, on the choice of $\mathcal{F}$, so $\bar{R} J(k, A)$ is a particular representative in the quasi-isomorphism class of $R J(k, A)$. By (3.5.1), $\bar{R} J(k, A)$ is a dg-manifold.

(3.7.3) Theorem. (a) We have $\pi_{0}(R J(k, A))=J(k, A)$.

(b) If $I \subset A$ is an ideal representing a $\mathbb{K}$-point $[I] \in J(k, A)$, then

$$
H^{i} T_{[I]}^{\bullet} R J(k, A)=\operatorname{Ext}_{A / I}^{i+1}\left(\mathbb{L}_{(A / I) / A}, A / I\right) .
$$

Proof. Part (a) is clear from the corresponding statements about the two constructions in (3.1). To prove (b), recall the transitivity triangle of relative cotangent complexes (see, e.g., Q , Th. 5.1) corresponding to the morphisms of rings $\mathbb{K} \rightarrow A \rightarrow A / I$ :

$$
(A / I) \otimes_{A} \mathbb{L}_{A / \mathbb{K}} \longrightarrow \mathbb{L}_{(A / I) / \mathbb{K}} \longrightarrow \mathbb{L}_{(A / I) / A} \longrightarrow(A / I) \otimes_{A} \mathbb{L}_{A / \mathbb{K}}[1] .
$$

This is a distinguished triangle in the derived category of complexes of $A / I$-modules. By applying the functor $\mathrm{RHom}_{A / I}(-, A / I)$, we get the triangle

$$
\operatorname{RHom}_{A / I}\left(\mathbb{L}_{(A / I) / A}, A / I\right) \rightarrow \operatorname{RHom}_{A / I}\left(\mathbb{L}_{(A / I) / \mathbb{K}}, A / I\right) \rightarrow \operatorname{RHom}_{A / I}\left(\mathbb{L}_{A / \mathbb{K}}, A / I\right),
$$

of which the first term has the cohomology appearing in the statement of (b). Notice that the other two terms are directly related with the derived versions of the two constructions of (3.1). Namely, Theorem 2.3.1 together with Proposition 3.5.2(b) imply that the middle term is quasi-isomorphic to the tangent space of $R C A(A / \tilde{V})$ at the point represented by $I$ and by the canonical algebra structure on $A / I$. Similarly, Proposition 3.6.4(b) implies that the right term is quasi-isomorphic to the tangent space to $R M_{f}$ at the same point as above. Notice further that the successive application of the two constructions $\left(R C A\right.$ and $\left.M_{f}\right)$ gives rise, at the level of tangent spaces, to the cone of a natural morphism of the tangent spaces to the $\mathrm{dg}$-schemes given by each construction separately. The theorem is proved.

(3.7.6) The graded case. Suppose that $A=\bigoplus A_{n}$ has an extra grading, as in (1.2). Then all the previous constructions are modified straightforwardly to give the derived scheme of graded ideals $R J(k, A)$, where $k=\left(k_{i}\right)$ is sequence of integers. As before, we use the notation $\bar{R} J(k, A)$ for the particular model obtained by using the resolutions mentioned in Definition 3.7.2.

In particular, for any graded ideal $I \subset A$ representing a point $[I] \in J(k, A)$ we have

$$
H^{i} T_{I}^{\bullet} R J(k, A)=\operatorname{Ext}_{A / I}^{i+1,0}\left(\mathbb{L}_{(A / I) / A}, A / I\right),
$$

where $\operatorname{Ext}^{i+1,0}$ is the degree 0 component of Ext ${ }^{i+1}$. 
(3.8) The relative version. Let $S$ be a scheme over $\mathbb{K}$ and let $\mathcal{A}$ be a sheaf of commutative $\mathcal{O}_{S}$-algebras (possibly without unit) such that as a sheaf of $\mathcal{O}_{S}$-modules, $\mathcal{A}$ is locally free of finite rank. Then the construction of $\bar{R} J(k, A)$ relativizes immediately, giving the relative derived ideal scheme $\bar{R} J(k, \mathcal{A} / S)$ which is a dg-scheme equipped with a smooth morphism to $S$. To construct it, we first form the relative Grassmannian $G(k, \mathcal{A} / S) \rightarrow S$, which is equipped with the tautological quotient bundle $\mathcal{A} / \widetilde{V}$, and then form $R C A(\mathcal{A} / \widetilde{V})$, the derived space of commutative algebra structures in the fibers of $\mathcal{A} / \widetilde{V}$ and the derived homomorphicity locus of the natural morphism of sheaves of (homotopy) commutative algebras on it, as in (3.7). By construction (and by the naturality and the tensor nature of the resolutions $\Lambda$ for the operad $\mathcal{C}$ om and $\mathcal{L}(\mathcal{A})$ for the $\mathcal{O}_{S}$-Algebra $\mathcal{A}$ ), we have the following compatibility statement.

(3.8.1) Proposition. (a) We have $\pi_{0} R J(k, \mathcal{A} / S)=J(k, \mathcal{A} / S)$; see (1.4).

(b) Let $\phi: S^{\prime} \rightarrow S$ be a morphism of schemes and $\mathcal{A}$ a sheaf of commutative $\mathcal{O}_{S}$-algebras, which is locally free as a sheaf of $\mathcal{O}_{S}$-modules. Then $\bar{R} J\left(k,\left(\phi^{*} \mathcal{A}\right) / S^{\prime}\right)$ is isomorphic to the fiber product of $\bar{R} J(k, \mathcal{A} / S)$ and $S^{\prime}$ over $S$.

See [CK], Sect. (2.8), for the definition of the fiber products in the dg-category. Note that we consider here the "straight" fiber product (which in our situation is quasi-isomorphic to the derived one).

Further, if $\mathcal{A}$ is equipped with an extra grading $\mathcal{A}=\bigoplus \mathcal{A}_{n}$, as in (1.2) and (3.7.6) (so that each $\mathcal{A}_{n}$ is an $\mathcal{O}_{S}$-submodule in $\mathcal{A}$ ) and $k=\left(k_{i}\right)$ is a sequence of integers, then we have the relative derived scheme of graded ideals $\bar{R} J(k, \mathcal{A} / S)$ defined in an obvious way.

\section{The DeRIVed Hilbert SCHEME}

(4.1) Graded ideals in truncations of the coordinate ring. We return to the situation of (1.3), so $A=\bigoplus_{i} A_{i}$ is the coordinate algebra of a projective variety $X \subset \mathbb{P}^{n}$.

We consider the finite-dimensional graded algebra $A_{[p, q]}$ and the corresponding derived scheme of graded ideals $R J\left(k, A_{[p, q]}\right)$. By Theorem 1.3.1, for $0 \ll p \ll q$ we have $\pi_{0} R J\left(k, A_{[p, q]}\right)=\operatorname{Hilb}_{h}(X)$. We would like to show that the dg-structure on $R J\left(k, A_{[p, q]}\right)$ is "asymptotically correct". More precisely, let $Z \subset X$ be a closed subscheme with Hilbert polynomial $h$, let $\mathcal{I} \subset \mathcal{O}_{X}$ be its sheaf of ideals and let $I=\operatorname{Mod}(\mathcal{I})$ be the corresponding graded ideal in $A$. We will prove the following fact.

(4.1.1) Theorem. For $i>0$, for $p$ large enough (depending on $i$ ) and $q$ large enough (depending on $i, p$ ) and every $Z \in \mathrm{Hilb}_{h}(X)$ as before we have

$$
\operatorname{Ext}_{\mathcal{O}_{Z}}^{i}\left(\mathbb{L}_{Z / X}, \mathcal{O}_{Z}\right)=\operatorname{Ext}_{A_{[p, q]}^{i, 0} / I_{[p, q]}}\left(\mathbb{L}_{\left(A_{[p, q]} / I_{[p, q]}\right) / A_{[p, q]}}, A_{[p, q]} / I_{[p, q]}\right) .
$$

When $A$ varies in a flat family over a scheme $S$ of finite type, then numbers $p, q$ can be chosen so that the above conditions hold uniformly for all $\mathbb{K}$-points of $S$.

(4.1.2) Lemma. For any $i>0$, for $p$ large enough (depending on $i$ ), and any $Z \in \operatorname{Hilb}_{h}(X)$ we have

$$
\operatorname{Ext}_{\mathcal{O}_{Z}}^{i}\left(\mathbb{L}_{Z / X}, \mathcal{O}_{Z}\right)=\operatorname{Ext}_{A_{\geq p} / I_{\geq p}}^{i, 0}\left(\mathbb{L}_{\left(A_{\geq p} / I_{\geq p}\right) / A_{\geq p}}, A_{\geq p} / I_{\geq p}\right) .
$$


Proof. First, consider two coherent sheaves $\mathcal{F}, \mathcal{G}$ on $Z$ and let $M, N$ be the corresponding graded $A / I$-modules. Then Serre's theorem $\underline{\mathrm{Se}}$ implies that

$$
\operatorname{Ext}_{\mathcal{O}_{Z}}^{i}(\mathcal{F}, \mathcal{G})=\lim _{p} \operatorname{Ext}_{A / I}^{i, 0}\left(M_{\geq p}, N_{\geq p}\right),
$$

and that this limit is achieved and, moreover, achieved uniformly if $Z, \mathcal{F}, \mathcal{G}$ run in a flat family over a scheme of finite type. We claim that, further, for $p \gg 0$ (with the same uniformity conditions),

$$
\operatorname{Ext}_{A / I}^{i, 0}\left(M_{\geq p}, N_{\geq p}\right)=\operatorname{Ext}_{A_{\geq p} / I_{\geq p}}^{i, 0}\left(M_{\geq p}, N_{\geq p}\right) .
$$

Indeed, in the case $i=0$ (when we are dealing with Hom), this is obtained by using the trick with the Veronese subalgebra from the proof of Theorem 1.3.2. The case $i>0$ is formally deduced from this by applying the derived functor.

We now specialize to the case when $\mathcal{G}=\mathcal{O}_{Z}$ and $\mathcal{F}$ is one of the first $i$ cohomology sheaves of the complex $\mathbb{L}_{Z / X}^{\bullet}$. Since, by Theorem 2.2.2, $\operatorname{Sh}\left(\mathbb{L}_{(A / I) / A}\right)=\mathbb{L}_{Z / X}$, the lemma follows by a spectral sequence argument.

In virtue of the lemma, Theorem 4.1.1 would be implied by the following general proposition applied to the canonical morphism $A_{\geq p} \rightarrow A_{\geq p} / I_{\geq p}$.

(4.1.3) Proposition. Let $f: A \rightarrow B$ be a morphism of finitely generated commutative graded algebras, situated in projective degrees $\geq 0$ (and cohomological degree 0 ). Then for each $i$ and large enough $q$ (depending on $i$ ) we have

$$
\operatorname{Ext}_{B}^{i, 0}\left(\mathbb{L}_{B / A}^{\bullet}, B\right)=\operatorname{Ext}_{B \leq q}^{i, 0}\left(L_{B_{\leq q} / A_{\leq q}}^{\bullet}, B \leq q\right) .
$$

Further, for fixed $i$ one can choose $q$ uniformly, if $A, B, f$ vary in a flat family over a scheme of finite type.

Proof. For a bigraded dg-algebra $C$ and a dg-module $M$ over $C$ we write

$$
\operatorname{RDer}(C, M)=\operatorname{RHom}_{C}\left(\mathbb{L}_{C / \mathbb{K}}^{\bullet}, M\right) .
$$

Note that if $C$ has trivial cohomological grading, then

$$
H^{0} \operatorname{RDer}(C, M)=\operatorname{Der}(C, M)
$$

is the space of derivations $C \rightarrow M$. We will use the notation $\operatorname{Der}(C, M)$ to signify the space of derivations for arbitrary $C, M$ as well.

Returning to the situation of $f: A \rightarrow B$, we have the transitivity triangle similar to (3.7.4), which we write in the form

$$
\operatorname{RHom}_{B}\left(\mathbb{L}_{B / A}^{\cdot}, B\right) \rightarrow \operatorname{RDer}(B, B) \rightarrow \operatorname{RDer}(A, B) .
$$

We have a similar triangle for the truncated morphisms $A_{\leq q} \rightarrow B_{\leq q}$. Thus our statement follows from the next proposition.

(4.1.4) Proposition. Let $M$ be a finitely generated B-module (situated in cohomological degree 0$)$. Then for each $i$ and large enough $q$ (depending on $i$ ) we have

$$
H^{i, 0} \operatorname{RDer}(B, M)=H^{i, 0} \operatorname{RDer}\left(B_{\leq q}, M_{\leq q}\right),
$$

and $q$ can be chosen uniformly for a flat family of $B, M$ over a scheme of finite type. 
Proof. Let $F \rightarrow B$ be a quasi-free resolution. Thus $F$ is a bigraded dg-algebra situated in cohomological degrees $\leq 0$ and we can assume that $F$ has finitely many generators in each cohomological degree. By definition,

$$
\operatorname{RDer}(B, M)=\operatorname{Der}(F, M) .
$$

We first prove the following fact.

(4.1.5) Lemma. For each $i$ and for $q \gg 0$ (depending on $i$ ) we have

$$
H^{i, 0} \operatorname{Der}(F, M)=H^{i, 0} \operatorname{Der}\left(F_{\leq q}, M_{\leq q}\right) .
$$

Proof. A derivation $D: F \rightarrow M$ is uniquely determined by its values on the generators of $F$, which can be chosen in an arbitrary way. So if we choose $q$ to be greater than the projective degrees of the generators of $F$ in cohomological degrees $0,-1, \ldots,-i-1$, then the complex $\operatorname{Der}^{0}(F, M)$ of derivations preserving the projective degrees coincides, in degrees $\leq i+1$, with the similar $\operatorname{complex} \operatorname{Der}^{0}\left(F_{\leq q}, M_{\leq q}\right)$ and hence the two complexes have the same cohomology in degrees $0,1, \ldots, i$. The uniformity in this situation is clear.

(4.1.6) Lemma. For each $i, q$ we have

$$
H^{i, 0} \operatorname{Der}\left(F_{\leq q}, M_{\leq q}\right)=H^{i, 0} \operatorname{RDer}\left(F_{\leq q}, M_{\leq q}\right) .
$$

Proof. To find $\operatorname{RDer}\left(F_{\leq q}, M_{\leq q}\right)$, we need a quasi-free resolution $G$ of $F_{\leq q}$. To construct such a $G$, we start from $F$ and then add new generators to $F$ so as, first, to make all the cocycles of $F$ in projective degrees $>q$ into coboundaries and then, inductively, to similarly kill any new cocycles that arise. In this way we get a factorization

$$
F \hookrightarrow G \stackrel{\text { qis }}{\longrightarrow} F_{\leq q},
$$

and any new generator which is added in forming $G$ starting from $F$ is in projective degree $>q$. This means that

$$
\operatorname{RDer}\left(F_{\leq q}, M_{\leq q}\right):=\operatorname{Der}\left(G, M_{\leq q}\right)=\operatorname{Der}\left(F, M_{\leq q}\right)=\operatorname{Der}\left(F_{\leq q}, M_{\leq q}\right),
$$

because any of the new generators must necessarily go to 0 in $M_{\leq q}$ by (projective) degree reasons. This proves the lemma. Proposition 4.1.4 follows.

This finishes the proof of Theorem 4.1.1.

(4.2) The approximations to RHilb. We continue to work in the situation of (4.1) and would like to use the dg-manifolds $R J\left(k, A_{[p, q]}\right)$ as approximations to the derived Hilbert scheme.

Let $X=\left(X^{0}, \mathcal{O}_{X}^{\bullet}\right)$ be a dg-scheme, so that we have the sheaf of graded algebras $\underline{H}^{\bullet}\left(\mathcal{O}_{X}^{\bullet}\right)$ on $\pi_{0}(X)=\operatorname{Spec} \underline{H}^{0}\left(\mathcal{O}_{X}\right)$. Let $\underline{H}^{\geq-m}\left(\mathcal{O}_{X}^{\bullet}\right), m \geq 0$, denote the truncation of this sheaf obtained by disregarding the cohomology in degrees $<-m$. We denote by $X_{h}^{\leq m}$ the graded scheme $\left(\pi_{0}(X), \underline{H}^{\geq-m}\left(\mathcal{O}_{X}^{\bullet}\right)\right)$. We call a morphism $f: X \rightarrow Y$ of dg-schemes an $m$-quasi-isomorphism if the induced morphism of graded schemes $f_{h}^{\leq m}: X_{h}^{\leq m} \rightarrow Y_{h}^{\leq m}$ is an isomorphism.

(4.2.1) Proposition. Let $f: M \rightarrow N$ be a morphism of dg-manifolds. Then the following are equivalent:

(i) $f$ is an m-quasi-isomorphism. 
(ii) $\pi_{0}(f): \pi_{0}(M) \rightarrow \pi_{0}(N)$ is an isomorphism and for any field extension $\mathbb{F} \supset \mathbb{K}$ and any $\mathbb{F}$-point $x \in M$ the differential $d_{x} f$ induces isomorphisms $\pi_{i}(M, x) \rightarrow$ $\pi_{i}(N, f(x))$ for $i \geq-m$.

Recall that $\pi_{i}(M, x)=H^{-i}\left(T_{x}^{\bullet} M\right)$. The proposition is proved in the same way as Proposition 2.5.9 of [CK].

We denote by $\mathcal{D} \leq m$ Man the category obtained from the category of dg-manifolds by formally inverting all the $m$-quasi-isomorphisms. Isomorphism classes of objects in $\mathcal{D} \leq m \mathcal{M}$ an can thus be seen as analogous to $m$-homotopy types in topology.

Returning to our particular situation, note that for any $p<q$ we have the morphisms of dg-manifolds

$$
\begin{aligned}
& \alpha_{p, q}: R J\left(k, A_{[p, q]}\right) \rightarrow R J\left(k, A_{[p+1, q]}\right), \\
& \beta_{p, q}: R J\left(k, A_{[p, q]}\right) \rightarrow R J\left(k, A_{[p, q-1]}\right),
\end{aligned}
$$

given by the natural projection (forgetting one of the graded components). These morphisms commute with each other in the obvious sense. Now, the results of (4.1) can be reformulated as follows.

(4.2.2) Proposition. Let $m \geq 0$ be given. Then there exists $p_{0} \geq 0$ such that for each $p \geq p_{0}$ there exists $q_{0}$ such that for each $q \geq q_{0}$ the morphisms $\alpha_{p, q}$ and $\beta_{p, q}$ are $m$-quasi-isomorphisms.

Proof. This follows from the equality (3.7.7), Theorem 4.1.1 and Proposition 4.2.1.

(4.2.3) Definition. The $m$-truncated derived Hilbert scheme $R H i l b b_{h}^{\leq m}(X)$ is the object of $\mathcal{D} \leq m$ Man represented by any of the dg-manifolds $R J\left(k, A_{[p, q]}\right)$ where $p, q$ are in the range given by Proposition 4.2.2.

Theorem 4.1.1 then implies the following

(4.2.4) Theorem. We have $\pi_{0} R_{H i l b}^{\leq m}(X)=H_{h} i b_{h}(X)$ and for each subscheme $Z \subset X$ with sheaf of ideals $\mathcal{I} \subset \mathcal{O}_{X}$ representing a $\mathbb{K}$-point $[Z] \in H_{i l b}(X)$,

$$
H^{i} T_{[Z]}^{\bullet} R H i l b_{h}^{\leq m}(X)=\operatorname{Ext}_{\mathcal{O}_{Z}}^{i}\left(\mathbb{L}_{Z / X}^{\bullet}, \mathcal{O}_{Z}\right), \quad 0 \leq i \leq m .
$$

(4.3) The case of locally complete intersections. In general, if $\mathbb{L}_{Z / X}^{\bullet}$ has infinitely many cohomology sheaves, none of the above truncations is sufficient to capture all the Ext's at once. We now concentrate on a special case when such uniform truncation is possible. Consider the open subscheme $\operatorname{Hilb}_{h}^{L C I}(X) \subset$ $H_{i l b}(X)$ formed by subschemes $Z \subset X$ with the Hilbert polynomial $h$, for which the embedding $Z \hookrightarrow X$ is a locally complete intersection morphism [I]. As is well known, for such $Z$ the relative cotangent complex is quasi-isomorphic to one sheaf in degree $(-1)$ which is locally free and called the conormal bundle:

$$
\mathbb{L}_{Z / X}^{\bullet}=N_{Z / X}^{*}[1]
$$

(In particular, if $X, Z$ are smooth, then we have (4.3.1) with $N_{Z / X}^{*}$ being the conormal bundle in the usual sense.) Therefore, for such $Z$,

$$
\operatorname{Ext}_{\mathcal{O}_{Z}}^{i}\left(\mathbb{L}_{Z / X}^{\bullet}, \mathcal{O}_{Z}\right)=H^{i-1}\left(Z, N_{Z / X}\right),
$$

and this vanishes whenever $i \geq \operatorname{dim}(Z)$.

Recall that for any dg-scheme $X=\left(X^{0}, \mathcal{O}_{X}^{\bullet}\right)$ and any open subset $U \subset X^{0}$ we have the induced dg-scheme $X_{U}=\left(U,\left.\mathcal{O}_{X}^{\bullet}\right|_{U}\right)$. 
Let $0 \ll p \ll q$ and let $U_{p, q} \subset R J\left(k, A_{[p, q]}\right)^{0}$ be the open subset obtained by removing the closed subset in $\operatorname{Hilb}_{h}(X)=\pi_{0}\left(R J\left(k, A_{[p, q]}\right)\right) \stackrel{\text { closed }}{\longleftrightarrow} R J\left(k, A_{[p, q]}\right)^{0}$ which is the complement of $\operatorname{Hilb}_{h}^{L C I}(X)$. The vanishing of sufficiently high Ext's in (4.3.2) gives us the following.

(4.3.3) Proposition. For $0 \ll p \ll q$ the dg-manifolds $R J\left(k, A_{[p, q]}\right)_{U_{p, q}}$ belong to the same quasi-isomorphism class (the quasi-isomorphisms being established by the appropriate restrictions of the morphisms $\alpha_{p, q}$ and $\left.\beta_{p, q}\right)$.

We denote $R_{H i l b}^{L C I}(X)$ and call the derived LCI-Hilbert scheme of $X$ the dgmanifold represented (up to quasi-isomorphism) by any of the $R J\left(k, A_{[p, q]}\right)_{U_{p, q}}$ above. If we need to emphasize the dependence of this manifold of $p$ and $q$, we will use the notation $\operatorname{RHilb}_{h}^{L C I}(X,[p, q])$. The following properties are now obvious from the construction.

(4.3.4) Theorem. We have $\pi_{0} \operatorname{RHilb}_{h}^{L C I}(X)=\operatorname{Hilb}_{h}^{L C I}(X)$ and for any locally complete intersection $Z \subset X$ representing a $\mathbb{K}$-point $[Z] \in H_{i l b}^{L C I}(X)$

$$
H^{i} T_{[Z]}^{\bullet} R H i l b_{h}^{L C I}(X)=H^{i}\left(Z, N_{Z / X}\right) .
$$

(4.3.5) Example: the derived space of maps. Let $C, Y$ be projective schemes with $Y$ being smooth. The scheme $\operatorname{Map}(C, Y)$ of maps $C \rightarrow Y$ can be seen as an open subscheme of the full Hilbert scheme $\operatorname{Hilb}(C \times Y)$ consisting of subschemes $C^{\prime} \subset C \times Y$ whose projection to $C$ is an isomorphism. This scheme is typically an infinite disjoint union of schemes of finite type. If we fix a projective embedding of $C \times Y$, then for any $h \in \mathbb{Q}[t]$ the intersection

$$
\operatorname{Map}_{h}(C, Y)=\operatorname{Map}(C, Y) \cap H_{i l b}(C \times Y)
$$

is a scheme of finite type which is open and closed in $\operatorname{Map}(C, Y)$. We can think of $h$ as being the "generalized degree" of a map $f: C \rightarrow Y$. Note that $\operatorname{Map}_{h}(C, Y) \subset$ $\operatorname{Hilb}_{h}^{L C I}(C \times Y)$.

Let $X=C \times Y$. Deleting from $R H i l b b_{h}^{L C I}(X,[p, q])^{0}$ the closed subset formed by points in $\operatorname{Hilb}_{h}^{L C I}(X)$ which do not represent graphs of maps, we get, by restriction, a dg-manifold $\operatorname{RMap}_{h}(C, Y)$ with the following properties:

$$
\begin{gathered}
\pi_{0} \operatorname{RMap}_{h}(C, Y)=\operatorname{Map}_{h}(C, Y) ; \\
H^{i} T_{[f]}^{\bullet} \operatorname{RMap}_{h}(C, Y)=H^{i}\left(C, f^{*} T Y\right), \quad i \geq 0 .
\end{gathered}
$$

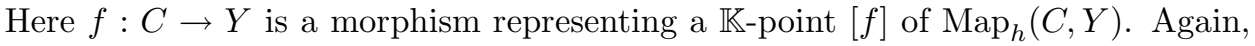
this dg-manifold itself depends, strictly speaking, on the choice of $0 \ll p \ll q$ and we will use the notation $\operatorname{RMap}_{h}^{[p, q]}(C, Y)$ if we will need to emphasize this dependence.

(4.4) The relative version. Let $S$ be a scheme over $\mathbb{K}$ and let $\pi: X \rightarrow S$ be a flat projective morphism. We fix an $S$-embedding $X \hookrightarrow S \times \mathbb{P}^{n}$ and denote by $\mathcal{O}(1)$ the corresponding relative very ample sheaf. We then have the sheaf of graded commutative algebras $\mathcal{A}=\bigoplus_{i} \mathcal{A}_{i}$ with $\mathcal{A}_{i}=\pi_{*} \mathcal{O}(i)$ being coherent and locally free for $i \gg 0$. Thus for $0 \ll p \ll q$ the dg-scheme $\bar{R} J\left(k, \mathcal{A}_{[p, q]} / S\right)$ from (3.8) is defined and is smooth over $S$. We also have the relative versions of the morphisms $\alpha_{p, q}$ and $\beta_{p, q}$ from (4.2) which we denote by the same letters.

(4.4.1) Proposition. Suppose $S$ is of finite type and let $m \geq 0$ be given. Then there exists $p_{0} \geq 0$ such that for each $p \geq p_{0}$ there exists $q_{0}$ such that for each $q \geq q_{0}$ the relative morphisms $\alpha_{p, q}$ and $\beta_{p, q}$ are m-quasi-isomorphisms. 
Proof. As in the proof of Proposition 4.2.2, we use the equality (3.7.7), Theorem 4.1.1 and Proposition 4.2.1. In addition, we use Proposition 3.8.1 to get the information about the relative tangent dg-spaces to $\bar{R} J\left(k, \mathcal{A}_{[p, q]} / S\right)$ at arbitrary $\mathbb{K}$-points.

We now define the relative $m$-truncated derived Hilbert scheme $R H i l b{ }_{h}^{\leq m}(X / S)$ to be the object of $\mathcal{D}^{\leq m} \mathcal{M}$ an represented by any of the dg-schemes $\bar{R} J\left(k, \mathcal{A}_{[p, q]} / S\right)$ where $p, q$ are in the range given by Proposition 4.4.1. Further, the definition of the relative derived LCI-Hilbert scheme $\operatorname{RHilb}_{h}^{L C I}(X / S)$ is completely analogous to that given in (4.3) and is left to the reader. By construction and by Theorem 1.4.1 we have

$$
\pi_{0} \operatorname{RHilb}_{h}^{\leq m}(X / S)=\operatorname{Hilb}_{h}(X / S), \quad \pi_{0} \operatorname{RHilb}_{h}^{L C I}(X / S)=\operatorname{Hilb}_{h}^{L C I}(X / S)
$$

as well as a compatibility statement analogous to Proposition 3.8.1(b).

We will use the notation $R H i l b^{L C I}(X / S,[p, q])$ if we need to emphasize the dependence on the choice of $p, q$.

Let $S$ be a scheme of finite type and let $C, Y$ be flat projective schemes over $S$ with $Y$ smooth. Then we have the relative derived space of $\operatorname{maps} \operatorname{RMap}_{h}(C, Y \mid S)$ (or $\operatorname{RMap}_{h}^{[p, q]}(C, Y \mid S)$, if we need to emphasize the dependence on the choice of $0 \ll p \ll q)$ defined, as in Example 4.3.5, via the relative derived LCI-Hilbert scheme $\operatorname{RHilb}_{h}^{L C I}\left(C \times_{S} Y,[p, q]\right)$. The following fact which we record for future reference, follows directly from the constructions and Proposition 3.8.1.

(4.4.2) Proposition. Let $\phi: S^{\prime} \rightarrow S$ be a morphism of schemes of finite type and let $C_{S^{\prime}}, Y_{S^{\prime}}$ be the pullbacks of $C, Y$ to schemes over $S^{\prime}$. If $0 \ll p \ll q$ are admissible for $S, C, Y$, then they are admissible for $S^{\prime}, C_{S^{\prime}}, Y_{S^{\prime}}$ as well and

$\operatorname{RMap}_{h}^{[p, q]}\left(C_{S^{\prime}}, Y_{S^{\prime}} \mid S^{\prime}\right)$ is isomorphic to the fiber product of $S^{\prime}$ and $\operatorname{RMap}_{h}^{[p, q]}(C, Y \mid S)$ over $S$.

\section{THE RELATIVE VERSION OVER A STACK AND DERIVED MODULI STACKS OF STABLE MAPS}

In this section we further relativize the above constructions to the case when the base scheme $S$ in (3.8) and (4.4) is replaced by an algebraic stack of finite type. As an application, we construct the derived stacks of stable maps, thus realizing the suggestion of Kontsevich [Kon].

Our basic reference for stacks is [LM] from which we adopt all the terminology and conventions. As before, we work over a field $\mathbb{K}$ of characteristic 0 .

(5.1) Dg-stacks. We first develop some minimal formalism necessary to speak about derived moduli stacks. While certainly too restrictive for all applications (e.g., for the construction of the derived moduli stack of vector bundles), this formalism will be sufficient for our problem.

(5.1.1) Definition. A dg-stack is a pair $\mathcal{X}=\left(\mathcal{X}^{0}, \mathcal{O}_{\mathcal{X}}^{\bullet}\right)$, where $\mathcal{X}^{0}$ is an algebraic

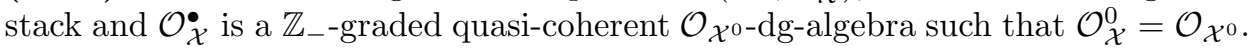
A graded stack is a dg-stack with trivial differential. 1- and 2-morphisms of dgstacks are defined as 1- and 2-morphisms of (dg-)ringed stacks ([LM], Def. 12.7.2).

We denote by $S t$ and $d g S t$ the 2-categories of algebraic stacks and dg-stacks respectively. 


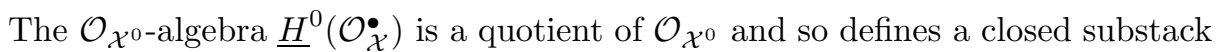

$$
\tau_{\leq 0} \mathcal{X}=\operatorname{Spec}\left(\underline{H}^{0}\left(\mathcal{O}_{\mathcal{X}}^{\bullet}\right) \subset \mathcal{X}^{0} .\right.
$$

See [LM], (14.2.7), for the meaning of Spec in this situation. It is clear that for any algebraic stack $\mathcal{Y}$ we have an equivalence of categories

$$
\operatorname{Hom}_{d g S t}(\mathcal{Y}, \mathcal{X}) \simeq \operatorname{Hom}_{S t}\left(\mathcal{Y}, \tau_{\leq 0} \mathcal{X}\right)
$$

Further, the sheaf of graded commutative algebras $\underline{H}^{\bullet}\left(\mathcal{O}_{\mathcal{X}}\right)$ is naturally a sheaf on $\tau_{\leq 0} \mathcal{X}$. We denote by $\mathcal{X}_{h}$ the graded stack $\left(\tau_{\leq 0} \mathcal{X}, \underline{H}^{\bullet}\left(\mathcal{O}_{\mathcal{X}}\right)\right)$. A 1-morphism of $\operatorname{dg}$-stacks $F: \mathcal{X} \rightarrow \mathcal{Y}$ is called a quasi-equivalence if $F_{h}: \mathcal{X}_{h} \rightarrow \mathcal{Y}_{h}$ is an equivalence of ringed stacks. The concept of an $m$-quasi-equivalence is defined similarly, by considering the truncations of $\underline{H}^{\bullet}\left(\mathcal{O}_{\mathcal{X}}^{\bullet}\right)$ and $\underline{H}^{\bullet}\left(\mathcal{O}_{\mathcal{Y}}^{\bullet}\right)$ in degrees $\geq-m$.

We will also denote by $\mathcal{X}_{\sharp}$ the graded stack $\left(\mathcal{X}^{0}, \mathcal{O}_{\mathcal{X}, \sharp}^{\bullet}\right)$, where $\mathcal{O}_{\mathcal{X}, \sharp}^{\bullet}$ is $\mathcal{O}_{\mathcal{X}}^{\bullet}$ with forgotten differential.

(5.1.2) Definition. A dg-stack $\mathcal{X}$ is said to be of (locally) finite type if $\mathcal{X}^{0}$ is an algebraic stack of (locally) finite type and each $\mathcal{O}_{\mathcal{X}}^{i}$ is a coherent $\mathcal{O}_{\mathcal{X}^{0} \text {-module. }}$

(5.1.3) Definition. Let $F: \mathcal{X} \rightarrow \mathcal{Y}$ be a 1-morphism of dg-stacks of finite type. We say that $F$ is smooth if, for any presentation (in the sense of [LM], Def. 4.14) of the morphism $F^{0}: \mathcal{X}^{0} \rightarrow \mathcal{Y}^{0}$

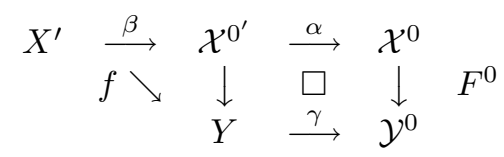

with $X^{\prime}, Y$ schemes of finite type, the morphism of dg-schemes of finite type

$$
\left(X^{\prime}, \beta^{*} \alpha^{*} \mathcal{O}_{\mathcal{X}}^{\bullet}\right) \rightarrow\left(Y, \gamma^{*} \mathcal{O}_{\mathcal{Y}}^{\bullet}\right)
$$

is smooth in the sense of [CK], Def. 2.7.1.

As usual, we say that $\mathcal{X}$ is smooth if $\mathcal{X} \rightarrow\{p t\}$ is a smooth morphism. Thus, in our terminology, every smooth dg-stack is of finite type.

(5.1.5) Definition. Let $\mathcal{X}$ be a smooth dg-stack, let $\mathbb{F}$ be a field extension of $\mathbb{K}$ and let $x$ be an $\mathbb{F}$-point of $\mathcal{X}$ (i.e., in fact an $\mathbb{F}$-point of $\tau_{\leq 0} \mathcal{X}$ ). The tangent dg-space $T_{x}^{\bullet} \mathcal{X}$ is a complex of $\mathbb{F}$-vector spaces situated in degrees $[-1, \infty)$ which is defined (up to a unique isomorphism in the derived category) as follows. Let $P: X^{0} \rightarrow \mathcal{X}^{0}$ be a presentation of $\mathcal{X}^{0}$ with $X^{0}$ a smooth algebraic variety, and let $X$ be the dg-manifold $\left(X^{0}, P^{*} \mathcal{O}_{\mathcal{X}}^{\bullet}\right)$. Then we set

$$
T_{x}^{\bullet} \mathcal{X}=\left\{\left(T_{X^{0} / \mathcal{X}^{0}}\right)_{x^{\prime}} \rightarrow T_{x^{\prime}}^{0} X \rightarrow T_{x^{\prime}}^{1} X \rightarrow \ldots\right\}
$$

where $x^{\prime} \in X^{0}$ is any $\mathbb{K}$-point over $x$ and $\left(T_{X^{0}} / \mathcal{X}^{0}\right)_{x^{\prime}}$ denotes the fiber at $x^{\prime}$ of the relative tangent bundle of $X^{0}$ over $\mathcal{X}^{0}$, which is embedded into $T_{x^{\prime}}^{0} X=T_{x^{\prime}} X^{0}$ in the standard way.

As in the case of dg-manifolds [CK], we denote

$$
\pi_{i}(\mathcal{X}, x)=H^{i} T_{x}^{\bullet} \mathcal{X}, \quad i=1,0,-1,-2, \ldots
$$


As in that case, we see easily that:

(5.1.6) Proposition. We have well-defined "Whitehead products"

$$
[-,-]: \pi_{i}(\mathcal{X}, x) \otimes \pi_{j}(\mathcal{X}, x) \rightarrow \pi_{i+j-1}(\mathcal{X}, x),
$$

making $\pi_{\bullet-1}(\mathcal{X}, x)$ into a graded Lie algebra. In particular, $\pi_{1}(\mathcal{X}, x)$ is a Lie algebra in the ordinary sense, and it is identified with the Lie algebra of the algebraic group $\operatorname{Aut}(x)$.

(5.1.7) Proposition. Let $F: \mathcal{X} \rightarrow \mathcal{Y}$ be a 1-morphism of smooth dg-stacks. Then the following are equivalent:

(i) $F$ is a quasi-equivalence (resp. an $m$-quasi-equivalence, $m \geq 0$ ).

(ii) The 1-morphism of algebraic stacks $\tau_{\leq 0} F: \tau_{\leq 0} \mathcal{X} \rightarrow \tau_{\leq 0} \mathcal{Y}$ is an equivalence and for any field extension $\mathbb{F} \supset \mathbb{K}$ and any $\mathbb{F}$-point $x$ of $\mathcal{X}$ the natural morphism of complexes $d_{x} F: T_{x}^{\bullet} \mathcal{X} \rightarrow T_{F(x)}^{\bullet} \mathcal{Y}$ is a quasi-isomorphism (resp. an m-quasiisomorphism).

Proof. This follows from the analogous result for dg-manifolds ([CK], Prop. 2.5.9) and the next obvious lemma applied to the morphism of sheaves $\left(\tau_{\leq 0} F\right)^{*} \underline{H}^{\bullet}\left(\mathcal{O}_{\mathcal{Y}}^{\bullet}\right)$ $\rightarrow \underline{H}^{\bullet}\left(\mathcal{O}_{\mathcal{X}}\right)$.

(5.1.8) Lemma. Let $\mathcal{Z}$ be an algebraic stack (of finite type) and let $f: \mathcal{F} \rightarrow \mathcal{G}$ be a morphism of quasi-coherent sheaves on $\mathcal{Z}$. Then $f$ is an isomorphism if and only if for any presentation $P: Z \rightarrow \mathcal{Z}$ (with $Z$ of finite type) the induced morphism $P^{*} \mathcal{F} \rightarrow P^{*} \mathcal{G}$ is an isomorphism.

(5.2) Dg-schematic local construction. We recall the formalism of [LM], $\S 14$, slightly modified for our purposes.

(5.2.1) Definition. Let $\mathcal{X}$ be an algebraic stack. A dg-schematic local construction on $\mathcal{X}$ is a datum $\underline{\mathcal{Y}}$ which associates a dg-scheme $\underline{\mathcal{Y}}_{U}$ to any morphism $U \rightarrow$ $X$ where $U$ is a scheme, and, further, associates an isomorphism of dg-schemes $V \times_{U} \underline{\mathcal{Y}}_{U}$ to any morphism $\phi: V \rightarrow U$ of schemes, so that these isomorphisms satisfy the cocycle conditions.

(5.2.2) Proposition. (a) Any dg-schematic local construction $\underline{\mathcal{Y}}$ on $\mathcal{X}$ gives rise to a dg-stack $\mathcal{Y}$ equipped with a morphism $\mathcal{Y} \rightarrow \mathcal{X}$ so that for any $U \rightarrow \mathcal{X}$ as above the dg-stack $U \times \mathcal{X} \mathcal{Y}$ is equivalent to the dg-scheme $\underline{\mathcal{Y}}_{U}$.

(b) Further, the algebraic stack $\tau_{\leq 0} \mathcal{Y}$ corresponds to the local construction $U \mapsto$ $\pi_{0} \underline{\mathcal{Y}}_{U}$

Proof. (a) When the dg-structures on the $\underline{\mathcal{Y}}_{U}$ are trivial, this is Proposition 14.1.7 of [LM] (with algebraic spaces replaced by schemes). One proves, in a similar way, a version of the cited statement in which the $\underline{\mathcal{Y}}_{U}$ are not just schemes but ringed schemes (i.e., schemes with a quasi-coherent sheave of rings) and, further, dg-ringed schemes. This implies (a). Part (b) is equally clear.

If $\mathcal{X}$ is a stack of finite type, then in the above discussion it is clearly sufficient to consider the schemes $U$ of finite type, which we will do.

(5.3) Derived spaces of maps over a stack. Let $\mathcal{S}$ be an algebraic stack of finite type and let $\mathcal{A}$ be a commutative $\mathcal{O}_{\mathcal{S}}$-algebra which, as an $\mathcal{O}_{\mathcal{S}}$-module, is locally free of finite rank. Fix $k \geq 0$. Application of the concept of a schematic local construction gives the relative Grassmannian $G(k, \mathcal{A} / \mathcal{S})$ and the relative ideal 
space $J(k, \mathcal{A} / \mathcal{S})$ which are algebraic stacks equipped with schematic projective morphisms to $\mathcal{S}$ with $J(k, \mathcal{A} / \mathcal{S})$ being a closed substack in $G(k, \mathcal{A} / \mathcal{S})$.

Notice further that associating to any morphism $\phi: U \rightarrow \mathcal{S}$ with $U$ a scheme, the dg-scheme $\bar{R} J\left(k,\left(\phi^{*} \mathcal{A}\right) / U\right.$ ) (see (3.8)) is a dg-schematic local construction in virtue of Proposition 3.8.1(b). Thus we get a dg-stack $\bar{R} J(k, \mathcal{A} / \mathcal{S})$ with $\tau_{\leq 0} \bar{R} J(k, \mathcal{A} / \mathcal{S})=$ $J(k, \mathcal{A} / \mathcal{S})$.

Similarly, if $\mathcal{A}=\bigoplus_{i=a}^{b} \mathcal{A}_{i}$ is a graded $\mathcal{O}_{\mathcal{S}}$-algebra with each $\mathcal{A}_{i}$ being locally free of finite rank as an $\mathcal{O}_{\mathcal{S}}$-module and $k=\left(k_{i}\right)_{i=a}^{b}$, we get the dg-stack of graded ideals which we still denote $\bar{R} J(k, \mathcal{A} / \mathcal{S})$.

Next, assume that $\mathcal{S}$ is of finite type, let $\pi: \mathcal{X} \rightarrow \mathcal{S}$ be a flat schematic projective morphism of algebraic stacks and let $\mathcal{O}(1)$ be the fixed relatively very ample sheaf on $\mathcal{X}$. Then setting $\mathcal{A}_{i}=\pi_{*} \mathcal{O}(i)$, we find that for $i \gg 0$ each $\mathcal{A}_{i}$ is locally free of finite rank as an $\mathcal{O}_{\mathcal{S}}$-module and so $\mathcal{A}_{[p, q]}=\bigoplus_{i=p}^{q} \mathcal{A}_{i}$ is an $\mathcal{O}_{\mathcal{S}}$-algebra satisfying the above conditions, so the dg-stack $\bar{R} J\left(k, \mathcal{A}_{[p, q]} / \mathcal{S}\right)$ is defined. Because $\mathcal{S}$ is of finite type, we see, by using Lemma 5.1.8, that for $0 \ll p \ll q$ the dg-stacks $\bar{R} J\left(k, \mathcal{A}_{[p, q]} / \mathcal{S}\right)$ have the same $m$-quasi-equivalence class and thus can be, with some abuse of notation, denoted by $R H i l b_{h}^{\leq m}(\mathcal{X} / \mathcal{S})$. By construction,

$$
\tau_{\leq 0} R \operatorname{Rilb}_{h}^{\leq m}(\mathcal{X} / \mathcal{S})=\operatorname{Hilb}_{h}(\mathcal{X} / \mathcal{S})
$$

is the relative Hilbert scheme of $\mathcal{X}$ over $\mathcal{S}$. Further, associating the dg-scheme $R H i l b_{h}^{L C I}\left(\phi^{*} \mathcal{X} / U,[p, q]\right)$ to any $\phi: U \rightarrow \mathcal{S}$ (see (4.2.4)) again forms a dg-schematic construction so we get a dg-stack $R H i l b_{h}^{L C I}(\mathcal{X} / \mathcal{S},[p, q])$ whose quasi-equivalence type for $0 \ll p \ll q$ is independent on the choice of such $p, q$.

Finally, let $\mathcal{C} \rightarrow \mathcal{S}, \mathcal{Y} \rightarrow \mathcal{S}$ be flat schematic projective morphisms, with $Y$ being smooth. By the same reasoning as before, we get the relative space of maps $R M a p_{h}^{[p, q]}(\mathcal{C}, \mathcal{Y} \mid \mathcal{S})$ whose quasi-equivalence type for $0 \ll p \ll q$ is independent on the choice of such $p, q$.

(5.4) The derived stack of stable maps. Let $\widetilde{M}_{g, n}$ be the stack of prestable $n$-pointed curves of genus $g$; see $[\mathrm{BM}], \S 2$. Thus an $S$-point of this stack is a system $\left(\pi: C \rightarrow S, x_{1}, \ldots, x_{n}\right)$, where $\pi$ is a flat proper morphism $\pi: C \rightarrow S$ of relative dimension 1 such that every geometric fiber $C_{s}, s \in S$, is a connected projective curve of arithmetic genus $g$, with at most nodal singularities, and where the $x_{i}: S \rightarrow C$ are sections whose values at every geometric point $s \in S$ are smooth and distinct points of the curve $C_{s}$. We denote by $\mathcal{C} \rightarrow \widetilde{M}_{g, n}$ the universal curve. This is a schematic projective 1-morphism of stacks.

If $K$ is a field and $\left(C, x_{1}, \ldots, x_{n}\right)$ is an $n$-pointed prestable curve of genus $g$ over $K$, we denote by $\left[C, x_{1}, \ldots, x_{n}\right]$ the corresponding $K$-point of $\widetilde{M}_{g, n}$. As for any $K$-point of any algebraic stack, the tangent space $T_{\left[C, x_{1}, \ldots, x_{n}\right]}^{\bullet} \widetilde{M}_{g, n}$ is a complex of $K$-vector spaces, situated in degrees $[-1,0]$ and defined up to quasi-isomorphism. The first order deformation theory gives an identification

$$
T_{\left[C, x_{1}, \ldots, x_{n}\right]}^{\bullet} \widetilde{M}_{g, n}=R \Gamma\left(C, R T C\left(-x_{1}-\ldots-x_{n}\right)\right)[1],
$$

where $R T C$ is the tangent complex of $C$.

(5.4.2) Definition. Let $(Y, \mathcal{O}(1))$ be a smooth projective variety over $\mathbb{K}$, let $d \in \mathbb{Z}$ and let $S$ be a $\mathbb{K}$-scheme. An $n$-pointed prestable map of genus $g$ and degree $d$ over $S$ is a system $\left(C, x_{1}, \ldots, x_{n}, f\right)$ where $\pi: C \rightarrow S$ and $x_{i}: S \rightarrow C$ are as in (5.1.1) 
while $f: C \rightarrow S \times Y$ is an $S$-map such that for any geometric point $s \in S$ the degree of the sheaf $f^{*} \mathcal{O}(1)$ on $C_{s}$ is $d$.

We denote by $\widetilde{M}_{g, n}(Y, d)$ the stack of prestable maps, as in (5.1.2). This is an algebraic stack which is nonseparated and possibly nonsmooth. By definition, we have the following; cf. [B], p. 604.

(5.4.3) Proposition. The stack $\widetilde{M}_{g, n}(Y, d)$ is naturally identified with the relative space of maps $\operatorname{Map}_{h}\left(\mathcal{C}, \widetilde{M}_{g, n} \times Y \mid \widetilde{M}_{g, n}\right)$ where $h \in \mathbb{Q}[t]$ is a polynomial uniquely determined by $g, d$ and the sheaf $\mathcal{O}(1)$ on $Y$.

As before, if $K$ is a field and $\left(C, x_{1}, \ldots, x_{n}, f\right)$ is an $n$-pointed prestable map over $K$, we denote by $\left[C, x_{1}, \ldots, x_{n}, f\right]$ the corresponding $K$-point of $\widetilde{M}_{g, n}(Y, d)$. The first order deformation theory now identifies

$$
\begin{gathered}
T_{\left[C, x_{1}, \ldots, x_{n}, f\right]}^{\bullet} \widetilde{M}_{g, n}(Y, d) \\
=\left(\tau_{\leq 1} R \Gamma\left(C, \text { Cone }\left\{R T C\left(-x_{1}-\ldots-x_{n}\right) \stackrel{d f}{\longrightarrow} f^{*} T Y\right\}\right)\right)[1],
\end{gathered}
$$

where $\tau_{\leq 1}$ is the cohomological truncation of a complex in degrees $\leq 1$. The $(-1)$ st cohomology of (5.4.4) is (as is the case with any algebraic stack) the Lie algebra of infinitesimal automorphisms of $\left(C, x_{1}, \ldots, x_{n}, f\right)$.

(5.4.5) Definition. A prestable map $\left(C, x_{1}, \ldots, x_{n}, f\right)$ over a field $\mathbb{F}$ is called stable if $H^{-1} T_{\left[C, x_{1}, \ldots, x_{n}, f\right]}^{\bullet} \widetilde{M}_{g, n}(Y, d)=0$. A prestable map over a $\mathbb{K}$-scheme $S$ is called stable if for any geometric point $s \in S$ the induced map over the field $\mathbb{K}(s)$ is stable.

We denote by $\bar{M}_{g, n}(Y, d) \subset \widetilde{M}_{g, n}(Y, d)$ the open substack formed by stable maps. It is known [Kon], $\mathrm{BM}$ that $\bar{M}_{g, n}(Y, d)$ is a proper Deligne-Mumford stack, in particular, it is separated and of finite type. However, it is not smooth in general, essentially because its tangent spaces, given by the same formula (5.4.4), are obtained by truncating some naturally arising complex. We now proceed to construct a smooth derived version of $\bar{M}_{g, n}(Y, d)$.

Forgetting the map $f$ defines morphisms of stacks

$$
\bar{\rho}: \bar{M}_{g, n}(Y, d) \rightarrow \widetilde{M}_{g, n}, \quad \widetilde{\rho}: \widetilde{M}_{g, n}(Y, d) \rightarrow \widetilde{M}_{g, n} .
$$

Note that although $\widetilde{M}_{g, n}$ is only locally of finite type, $\bar{M}_{g, n}(Y, d)$ is of finite type, so the image of $\bar{\rho}$ is contained in an open substack $\mathcal{S}$ of $\widetilde{M}_{g, n}$ of finite type.

(5.4.7) Definition. Let $\mathcal{S} \subset \widetilde{M}_{g, n}$ be an open substack of finite type. The derived stack of prestable maps of type $\mathcal{S}$ is defined to be

$$
R \widetilde{M}_{g, n}^{\mathcal{S}}(Y, d)=\operatorname{RMap}_{h}(\mathcal{C}, Y \times \mathcal{S} \mid \mathcal{S}),
$$

where $h$ is as in (5.4.3).

(5.4.8) Theorem. (a) $R \widetilde{M}_{g, n}^{\mathcal{S}}(Y, d)$ is a smooth dg-stack and $\tau_{\leq 0} R \bar{M}_{g, n}^{\mathcal{S}}(Y, d)=$ $\widetilde{\rho}^{-1}(\mathcal{S})$.

(b) If $\left(C, x_{1}, \ldots, x_{n}, f\right)$ is an n-pointed prestable map over $\mathbb{K}$ such that $\left(C, x_{1}, \ldots, x_{n}\right)$ $\in \mathcal{S}$, then

$$
T_{\left[C, x_{1}, \ldots, x_{n}, f\right]}^{\bullet} R \widetilde{M}_{g, n}^{\mathcal{S}}(Y, d)=R \Gamma\left(C, \text { Cone }\left\{R T C\left(-x_{1}-\ldots-x_{n}\right) \stackrel{d f}{\longrightarrow} f^{*} T Y\right\}\right)[1] .
$$


Proof. (a) Smoothness of $R \widetilde{M}_{g, n}^{\mathcal{S}}(Y, d)$ follows from the smoothness of $\mathcal{S}$ and from the relative smoothness of RMap. The identification of $\tau_{\leq 0}$ follows from Proposition 5.4.3 and from the identification $\pi_{0}$ RMap = Map.

(b) Let us write $R \widetilde{M}^{\mathcal{S}}$ for $R \widetilde{M}_{g, n}^{\mathcal{S}}(Y, d)$ and $x$ for $x_{1}, \ldots, x_{n}$. Then we have the distinguished triangle of complexes (relative vs. absolute tangent dg-spaces)

$$
T_{[C, x, f]}^{\bullet}\left(R \widetilde{M}^{\mathcal{S}} / \mathcal{S}\right) \rightarrow T_{[C, x, f]}^{\bullet} R \widetilde{M}^{\mathcal{S}} \rightarrow T_{[C, x]}^{\bullet} \mathcal{S} .
$$

Now, $T_{[C, x]}^{\bullet} \mathcal{S}$ is given by (5.4.1), while by Proposition 4.4.2

$$
T_{[C, x, f]}^{\bullet}\left(R \widetilde{M}^{\mathcal{S}} / \mathcal{S}\right)=T_{[f]}^{\bullet} \operatorname{RMap}_{h}(C, Y)=R \Gamma\left(C, f^{*} T Y\right)
$$

and our assertion follows from comparing the above triangle with the triangle

$$
R \Gamma\left(C, f^{*} T Y\right) \rightarrow R \Gamma(C, \text { Cone }) \rightarrow R \Gamma\left(C, R T C\left(-x_{1}-\ldots-x_{n}\right)\right)[1],
$$

where Cone is the cone of $d f$ in the formulation of (b). The theorem is proved.

For a dg-stack $\mathcal{X}$ and an open substack $\mathcal{U} \subset \mathcal{X}^{0}$ we have a dg-stack $\left.\mathcal{X}\right|_{\mathcal{U}}=$ $\left(\mathcal{U}, \mathcal{O}_{\mathcal{X}}^{\bullet} \mid \mathcal{U}\right)$. Further, since $\tau_{\leq 0} \mathcal{X}$ is a closed substack of $\mathcal{X}^{0}$, any open subset $\mathcal{V} \subset$ $\tau_{\leq 0} \mathcal{X}$ gives rise to an open substack $\mathcal{V}^{\prime} \subset \mathcal{X}^{0}$ which is the complement in $\mathcal{X}^{0}$ to the complement of $\mathcal{V}$ in $\tau_{\leq 0} \mathcal{X}$ considered as a closed substack in $\mathcal{X}$. Let us write $\mathcal{X}_{\mathcal{V}}$ for $\left.\mathcal{X}\right|_{\mathcal{V}^{\prime}}$.

(5.4.9) Definition. The derived moduli stack of stable maps is defined to be

$$
R \bar{M}_{g, n}(Y, d)=R \bar{M}_{g, n}^{\mathcal{S}}(Y, d)_{\bar{M}_{g, n}(Y, d)},
$$

where $\mathcal{S} \subset \bar{M}_{g, n}$ is any open substack containing the image of $\bar{\rho}$ in (5.4.6).

Definition 5.4.9 gives, in particular, a construction of the sheaf of graded commutative algebras $\underline{H}^{\bullet}\left(\mathcal{O}_{R M_{g, n}(Y, d)}^{\bullet}\right)$ on the Deligne-Mumford stack $\bar{M}_{g, n}(Y, d)$. It is this structure whose existence was originally conjectured by Kontsevich ([Kon, $\mathrm{n}$. 1.4.2) and used to give a formula for the virtual fundamental class (ibid., p. 344). In a subsequent paper we plan to study this fundamental class in more detail and, in particular, show that it coincides with the class constructed in $[\bar{B}],[\mathrm{BF}],[\mathrm{LT}]$.

\section{REFERENCES}

[B] K. Behrend, Gromov-Witten invariants in algebraic geometry, Invent. Math. 127 (1997), 601-617. MR 98i:14015

[BF] K. Behrend and B. Fantechi, The intrinsic normal cone, Invent. Math. 128 (1997), 45-88. MR 98e:14022

[BK] S. Barannikov and M. Kontsevich, Frobenius manifolds and formality of Lie algebras of polyvector fields, Internat. Math. Research Notices 4 (1998), 201-215. MR 99b:14009

$[\mathrm{BM}] \mathrm{K}$. Behrend and Y. Manin, Stacks of stable maps and Gromov-Witten invariants, Duke Math. J. 85 (1996), 1-60. MR 98i:14014

[CK] I. Ciocan-Fontanine and M. Kapranov, Derived Quot schemes, Ann. Sci. ENS (4) 34 (2001), 403-440.

[FP] W. Fulton and R. Pandharipande, Notes on stable maps and quantum cohomology, Proc. Sympos. Pure Math. 62 (1997), Pt. 2, p. 45-96. MR 98m:14025

[GJ] E. Getzler and J.D.S. Jones, Operads, homotopy algebras and iterated integrals for double loop spaces, preprint, 1994.

[GK] V. Ginzburg and M. Kapranov, Koszul duality for operads, Duke Math. J. 76 (1994), 203-272. MR 96a:18004

[Gr] A. Grothendieck, Techniques de construction et théorèmes d'existence en géometrie algébrique IV: Les schémas de Hilbert, Séminaire Bourbaki 221 (1960/61). MR 27:1339 
[Hi1] V. Hinich, Dg-coalgebras as formal stacks, J. Pure Appl. Algebra 162 (2001), 209-250. MR 2002f:14008

[Hi2] V. Hinich, Deformations of homotopy algebras, preprint math.AG/9904145.

[I] L. Illusie, Complexe Cotangent et Déformations (Lecture Notes in Math. 239), SpringerVerlag, 1972. MR 58:10886a

[Ka] M. Kapranov, Injective resolutions of $B G$ and derived moduli spaces of local systems, J. Pure Appl. Algebra 155 (2001), 167-179. MR 2002b:18017

[Kol] J. Kollar, Rational Curves in Algebraic Varieties, Springer-Verlag, 1996. MR 98c:14001

[Kon] M. Kontsevich, Enumeration of rational curves via torus actions, in: "Moduli Space of Curves" (R. Dijkgraaf, C. Faber, G. van der Geer Eds.) pp. 335-368, Birkhauser, Boston, 1995. MR 97d:14077

[KS] M. Kontsevich and Y. Soibelman, Deformations of algebras over operads and Deligne's conjecture, preprint math.QA/0001151.

[LM] G. Laumon and L. Moret-Bailly, Champs Algébriques, Springer-Verlag, 2000. MR 2001f:14006

[LT] J. Li and G. Tian, Virtual moduli cycles and Gromov-Witten invariants of algebraic varieties, J. of the AMS 11 (1998), 119-174. MR 99d:14011

[Lo] J.L. Loday, Cyclic Homology, Springer-Verlag, 1995. MR 94a:19004

[Man] M. Manetti, Extended deformation functors, I, Int. Math. Res. Not. 2002, no. 14, 719-756.

[Mar] M. Markl, Models for operads, Comm. Algebra 71 (1996), 1471-1500. MR 96m:18012

[May] J. P. May, Geometry of Iterated Loop Spaces (Lecture Notes in Math. 271), Springer-Verlag, 1972. MR 54:8623b

[Q] D. Quillen, On the (co)homology of commutative rings, Proc. Sympos. Pure Math. 17 (1970), 65-87. MR 41:1722

[Re] C. Rezk, Spaces of algebra structures and cohomology of operads, thesis, MIT (1996).

[Se] J.-P. Serre, Faisceaux algébriques cohérents, Ann. of Math. 61 (1955), 197-278. MR 16:953C

[St] J. D. Stasheff, Differential graded Lie algebras, quasi-Hopf algebras and higher homotopy algebras, Lecture Notes in Math. 1510 (1992), 120-137. MR 93j:17055

[Vi] E. Viehweg, Quasi-projective Moduli for Polarized Manifolds, Ergebnisse der Mathematik und ihrer Grenzgebiete (3), 30, Springer-Verlag, Berlin, 1995. MR 97j:14001

Department of Mathematics, University of Minnesota, Minneapolis, Minnesota 55455

E-mail address: ciocan@math.umn.edu

Department of Mathematics, University of Toronto, 100 St. George Street, Toronto, Ontario, CANADA M5S 3G3

E-mail address: kapranov@math.toronto.edu 